\title{
Modelling and Estimation for Uncertain Systems with Transmission Delays, Packet Dropouts, and Out-of-Order Packets
}

\author{
Li Liu $\mathbb{D}^{1,2}$ Aolei Yang, ${ }^{3}$ Wenju Zhou, ${ }^{1,3}$ Wasif Naeem, ${ }^{4}$ Gang Wang, ${ }^{1}$ and Hua Wang ${ }^{1}$ \\ ${ }^{1}$ School of Information Science and Electrical Engineering, Ludong University, 264025, Yantai, Shandong, China \\ ${ }^{2}$ Yantai Research Institute of New Generation Information Technology, Southwest Jiaotong University, 264025, Yantai, China \\ ${ }^{3}$ Shanghai Key Laboratory of Power Station Automation Technology, School of Mechatronic Engineering and Automation, \\ Shanghai University, 200072, Shanghai, China \\ ${ }^{4}$ School of Electronics, Electrical Engineering and Computer Science, Queens University Belfast, Belfast BT9 5AH, UK
}

Correspondence should be addressed to Li Liu; liulildu@163.com

Received 23 June 2018; Revised 21 September 2018; Accepted 2 October 2018; Published 18 October 2018

Guest Editor: Yimin Zhou

Copyright (C) $2018 \mathrm{Li}$ Liu et al. This is an open access article distributed under the Creative Commons Attribution License, which permits unrestricted use, distribution, and reproduction in any medium, provided the original work is properly cited.

\begin{abstract}
The study focuses on the modelling and estimation of a class of discrete-time uncertain systems, including network-induced random delays, packet dropouts, and out-of-order packets during the data transmission from the plant to the estimator. In order to improve system performance, event-triggered signal selection method is used to establish the system model. Based on this model, a distributed measurement and centralized fusion estimation scheme is designed using a robust finite horizon Kalman-type filter. Since the phenomena caused by the network-induced deteriorate estimation accuracy, a time-based reorganization measurement is employed to design a linear delay compensation strategy based on estimation. Moreover, in order to obtain the optimal linear estimation, weighted fusion estimation approach is used to perform information collaboration through the error cross-covariance matrix. Simulation results demonstrate that the proposed method has higher estimation performance than the existing methods in this study.
\end{abstract}

\section{Introduction}

The networked system is spatially distributed system based on shared communication technology $[1,2]$. They have attracted a large number of subjects of intensive researches and possessed the successful applications in a wide range, such as intelligent transportation [3], environmental monitoring [4], remote diagnostics and troubleshooting [5], and smart grids [6]. Note that the communication networks are introduced $[7,8]$ into distributed systems inevitably produce networkinduced phenomena, which usually deteriorate the performance of networked systems. Such network-induced phenomena include but are not limited to transmission delays, packet dropouts, missing/fading measurements, our-of-order packets, and variable sampling/transmission intervals [9]. Therefore, the research on distributed information perception and fusion estimation issue for networked systems with network-induced phenomena is significant and challenging $[10]$.

There are excellent results for the problem of state estimation. In engineering applications, stochastic uncertainties are described using the multiplicative noise [10-13] to simplify the system model. And the cross-correlation between process noise and measurement noise in $[11,14-16]$ is taken into account for data transmission over networks. In order to alleviate the negative influence of network-induced phenomena and improve system performance, some distributed estimation and filtering methods are proposed. Liu et al. [17] presented distributed fusion estimation based on Kalmantype filtering, which introduced two level weighted fusion scheme. For unknown nonlinear system, a robust adaptive finite-time parameter estimation [18] and control based on parameter estimation errors was studied. Furthermore, an adaptive law [19] is used to achieve precise estimation of 
essential parameters, where the parameter estimation error is obtained explicitly and then used as a new leakage term. Event-triggered communication schedules [20] are used for the distributed estimation problem employing Kalman consensus filter. Furthermore, filtering methods play an important role in networked systems for signal processing and communications. For example, event-based $H_{\infty}$ filtering $[7,21]$ is insensitive to the uncertainties appearing in stochastic system models and/or exogenous input signals. Considering the parametric uncertainty [22], robust filtering [23] has the robustness to suppress the noise disturbance. The finite-horizon filtering $[24,25]$ can obtain the upper bound to the steady-state error covariance. Using the discretetime stochastic bounded real lemma and the matrix analysis approach, Chen et al. [26] presented the distributed robust fusion estimation with stochastic and deterministic parameter uncertainties. Since the actual error covariance of estimated state is less than the upper bound, it has a better transient performance.

In order to deal with network-induced transmission delays and/or packet dropouts phenomena [27], there are some results available in the current investigations. The onestep prediction compensation scheme is used to describe the random delays, which applies the augmented state approach $[28,29]$ to obtain more accurate estimated value. For a class of networked multisensor fusion systems with multiple uncertainties, robust reduced-dimension observationfusion Kalman filters were proposed [30] to further reduce the computation burden. On the other hand, measurement reorganization $[24,31]$ is an effective strategy employing the measurement transformation method to transform the random delayed system into the delay-free counterpart. In addition, the system model is established by random variables of Bernoulli distribution $[1,13,28,32]$, so that the measurement model is augmented by multiple random delayed states. However, the compensation and state augmentation schemes increase the computational complexity of state estimation via networked systems.

For modelling problem, Brizhinev et al. [33] proposed a modelled method through the notions of power transition and power diffusion. Miao et al. [34] investigated the containment control of multi-agent systems with constant time delays under event-triggered conditions, and $\mathrm{Li}$ et al. [35] presented an event-triggered sampling mechanism and develops a sampled-data-based stabilizer for switching linear systems. Considering that random transmission delays inevitably generate out-of-order packets phenomenon in the communication, holding or dropping packet disorders is necessary to signal processing in networked systems. There are extensive reports on dealing with out-of-order packets. For example, to receive the most recent arrived data packet, the zero-order-holder $(\mathrm{ZOH})$ scheme is applied and the stored packet is replaced until the next arrived data packet $[36,37]$. The signal sequence reordering method of the logic $\mathrm{ZOH}[7,38-41]$ scheme is introduced a function based on $\mathrm{ZOH}$ to judge an out-of-order packet. So only the latest timestamped data packet is received. The scheme then actively drops the out-of-order packets. Note that logic $\mathrm{ZOH}$ is widely applied in networked control systems for event-trigger mechanism. However, with regard to the effect of out-oforder packets, the analysis and design of the finite-horizon filter based on event-triggered signal selection method are a complex problem, and it is difficult to detect the appropriate filter parameters.

Summarizing the above discussion, the estimation problem includes designing the filter with multi-step random delays, packet dropouts and correlated noises. However, for uncertain systems, the distributed fusion estimation based on finite horizon filtering are seldom studied. This may be due to the difficulty of dealing with the disordered and missing data packets, as well as probing the appropriate upper bounds for filter parameters.

Motivated by the above analysis, this study focuses on establishing system model and probing estimator of stochastic time-varying systems. For the received measurement with network-induced random transmission delays, packet dropouts, and out-of-order packets, these phenomena are synthetically considered; moreover, the system model is established by the signal selection method. The main contributions of this study are summarized as follows:

(i) Analyze the performance of time series signal processing for network-induced random transmission delays, packet losses, and disorders employing. Meanwhile, in order to effectively discard the out-of-order packets and improve system performance, the system model depending on the event-triggered signal selection method of logic $\mathrm{ZOH}$ is established for achieving state estimation.

(ii) The linear delay compensation strategy based on estimation is proposed for dealing with random transmission delays. In order to suppress the computational burden, the delayed estimation is transformed into the equivalent delayfree one by re-ordering measurement sequence with a timestamp.

(iii) Considering the delayed system with packet dropouts, the one-step prediction estimation approach is presented for compensating the missing packets with an artificial delay. Furthermore, the weighted fusion criterion is introduced using the distributed estimation based on each subsystem, and the fusion center is used to obtain more precise estimation accuracy than each local system.

The rest sections of this study are organized as follows. The uncertain system modelling problem is described in Section 2. In order to use the event-triggered signal to choose method, Section 3 designs distributed fusion estimation approach based on finite horizon robust Kalman-type filtering. Simulation results and analysis are given in Section 4 and the conclusions are outlined in Section 5.

Notations. Throughout this paper, the symbol $E(\cdot)$ denotes the mathematical expectation operator and the superscript $T$ is the transpose. $\mathbb{R}^{r}$ represents the $r$-dimensional Euclidean space, and $\mathbb{R}^{r \times r}$ denotes the set of all $r \times r$ real matrices. A real symmetric matrix $M>0$ expresses that $M$ is a positivedefinite matrix, $M^{-1}$ stands for the inverse of the positivedefinite matrix $M$, while $\operatorname{tr}(M)$ is the trace of matrix $M . \delta_{k, l}$ is the Kronecker function; i.e., $\delta_{k, l}=1$ if $k=l$; otherwise $\delta_{k, l}=0$ if $k \neq l$. 


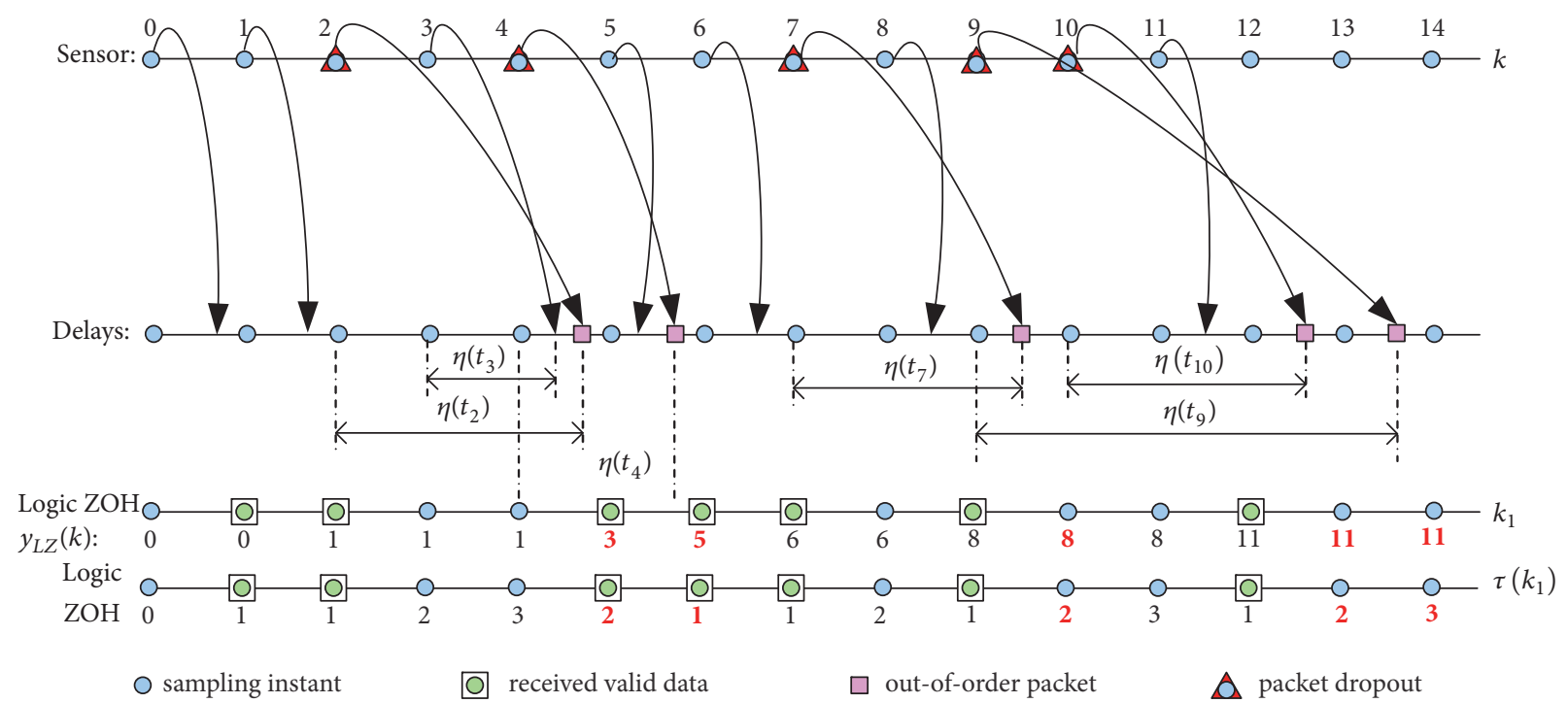

FIGURE 1: Sorting procedure for packet sequence based on event-triggered signal selection method.

\section{Problem Formulation and Analysis}

2.1. System Description. The considered uncertain system of each sensor is described by the following linear discrete-time system, which is investigated in $[10,13]$

$$
\begin{array}{r}
x(k+1)=\left(A_{k}+\mathscr{F}_{k} F_{k} E_{k}\right) x(k)+B_{k} w_{k}, \\
k=1,2, \cdots \\
z^{i}(k)=\left(C_{k}^{i}+\mathscr{H}_{k}^{i} F_{k} E_{k}^{i}\right) x(k)+v_{k}^{i}, \quad i=1, \cdots, L
\end{array}
$$

where $x(k) \in \mathbb{R}^{r}$ represents the state to be estimated and $z^{i}(k) \in \mathbb{R}^{m_{i}}$ expresses the measurement output of the $i$ th sensor at time instant $k . A_{k} \in \mathbb{R}^{r \times r}, B_{k} \in \mathbb{R}^{r}, C_{k}^{i} \in$ $\mathbb{R}^{m_{i} \times r}, \mathscr{F}_{k}, E_{k}, \mathscr{H}_{k}^{i}$ and $E_{k}^{i}$ are known time-varying matrices, and $F_{k}$ denotes the time-varying parameter uncertainties. $w_{k} \in \mathbb{R}$ and $v_{k}^{i} \in \mathbb{R}^{m_{i}}$ denote the process noise and measurement noise of the $i$-th sensor, which are zero mean white noise with covariance matrices $Q_{k}$ and $R_{k}^{i}$, respectively. The initial state $x(0)$ with mean $\mu_{0}$, and covariance $P_{0}$ is assumed to be uncorrelated with other noise signals. Note that the uncertainties $F_{k}$ satisfies $F_{k} F_{k}^{T} \leq I$.

\subsection{System Modelling Based on Event-Triggered Signal Selec-} tion Method. Taking the limited bandwidth into account, the data transmission from sensors to processors is limited and it would inevitably generate the network congestion. In this paper, the random transmission will inevitably bring packet drops and out-of-order packets. If the system model is established for each network-induced phenomenon, the system computation cost is significantly increased. Based on the abovementioned problem, network-induced phenomena are synthetically considered. Therefore, the event-triggered signal selection method of logic $\mathrm{ZOH}$ is designed to deal with packet dropouts and out-of-order packets.
Remark 1. For the time-stamped data packets in the network, the logic $\mathrm{ZOH}$ stores the latest data packet, and other time-stamped packets are discarded. It means that the stored data packets will be updated until the output of the logic $\mathrm{ZOH}$ being received to a latest signal. Because the latest data packet before being transmitted is close to the newest actual signal, the network-induced packet disorders during the transmission are dropped via the logic $\mathrm{ZOH}$ [38-40].

Without loss of generality, it is assumed that the measurement is sampled at a constant sample period $T$ for an arbitrary subsystem, and the sampling time instant is denoted by $t \in\{k T, k \in \mathbb{N}\}$. Set the largest transmission delays to be no more than $N$ steps, and $N(k) \leq k$ represents the largest data delays at time instant $k$. To further describe the networked systems in the presence of random transmission delays, packet dropouts, and out-of-order packets, a typical scenarios are represented in Figure 1.

Suppose that the upper boundary of time delays is no more than 5 sample periods (i.e., $N \leq 5$ ), wherein $\eta\left(t_{k}\right)$ denotes the network-induced transmission delays at time instant $k$. Figure 1 demonstrates the received valid data packets according to the logic $\mathrm{ZOH}$. From the time interval, there are three cases to be listed.

Case 1. At the same time interval, the arrived data packets with time-stamp contain out-of-order and in-order phenomena. When the sampling time $t \in[2 T, 5 T)$, the transmission delays are $\eta\left(t_{2}\right)=2 T+\eta^{\prime}\left(t_{2}\right)$ and $\eta\left(t_{3}\right)=1 T+\eta^{\prime}\left(t_{3}\right)$, respectively; here $\eta^{\prime}\left(t_{k}\right)$ is the transmission delay in one sample period. Due to $\eta^{\prime}\left(t_{2}\right)>\eta^{\prime}\left(t_{3}\right)$ shown in Figure 1, the out-of-order packets generate from signals $z(3)$ and $z(2)$ in the transmission. Note that $z(2)$ is the most recent data packet and $z(3)$ represents the latest packet. And then, signal $z(2)$ is discarded depending on the role of the logic $\mathrm{ZOH}$. 
Case 2. The arrived data packets with time-stamp are outof-order and in-order, at the neighboring time interval. For the sampling time $9 T$ and $10 T$, there exist the out-oforder packets, and the transmitted data are $z(8)$ and $z(7)$, respectively. Adopting the latest packets using the logic $\mathrm{ZOH}$, $z(7)$ is dropped at time instant 10T; meanwhile, the latest time-stamped data packet $z(8)$ is held.

Case 3. The time interval is more than one sampling period for the arrived out-of-order and in-order packets. At the sampling time $t \in[12 T, 14 T)$, the transmitted data are $z(11), z(10)$, and $z(9)$ sequentially, which are the most recent data package. However, signals $z(10)$ and $z(9)$ are dropped according to the criteria of receiving the latest data packet for the logic $\mathrm{ZOH}$.

As mentioned before, at the current time instant $k$, the time-stamp of the received valid data packet is denoted by $k_{1}$ and $k_{2}$ represents the time-stamp for the most recent transmission signal. The corresponding transmission delays from sensor to estimator are represented as $\eta\left(k_{1}\right)$ and $\eta\left(k_{2}\right)$ and satisfy $0 \leq \eta\left(k_{1}\right) \leq \eta\left(k_{2}\right) \leq N$. To clearly describe the time sequence, $\tau\left(k_{1}\right) \in \mathbb{N}$ and $\tau\left(k_{2}\right) \in \mathbb{N}$ denote the received data packet with delays using logic $\mathrm{ZOH}$ and random transmission delays at the sampling time, respectively, which satisfy

$$
k=\tau\left(k_{1}\right)+k_{1}=\tau\left(k_{2}\right)+k_{2}
$$

Take into account that the latest data packet is more approximate the current data packet, and an artificial variable $\beta(k) \geq 0$ is introduced to express the relationship between $k_{1}$ and $k_{2}$, that is,

$$
k_{1}=k_{2}+\beta(k)
$$

Note that the selected data packet with latest time-stamp is a relatively larger value than the data packet with random transmission delays. Without loss of generality, the delays are obtained from (3) and (4) i.e., $\tau\left(k_{1}\right)=\tau\left(k_{2}\right)-\beta(k)$ satisfies $\tau\left(k_{1}\right) \leq \tau\left(k_{2}\right)$. It implies that the data packet with less transmission delay is used to estimate the actual system state, improve the estimation accuracy, and further reduce the computation burden. Therefore, using logic $\mathrm{ZOH}$ scheme is more reasonable to deal with random transmission delays and further improve the system performance [39].

Remark 2. Adopting the signal selection method of logic $\mathrm{ZOH}$, the transmission signal with packet dropouts and out-of-order packets is transferred into random transmission delay with time-stamped data packets. To alleviate the computational burden, the measurement reorganization approach is investigated. When the logic $\mathrm{ZOH}$ receives the time-stamped data packet $z\left(k_{1}\right)$, the stored signal $y_{L Z}(k)$ is reorganized as

$$
y_{L Z}(k)=z\left(k-\tau\left(k_{1}\right)\right)
$$

It implies that when the time-stamped data packets transmit from a sensor to processor (or estimator) over a communication network, the processor (or estimator) is able to obtain the knowledge of packet delay and dropout value at every sampling time [24].
2.3. Cross-Correlated Noise. In the engineering applications, the distributed systems involve the cross-correlated noise [10, $13,28]$ during data transmission, which exist in a common noisy environment.

At the same time instant, it is assumed that the process noise $w_{k}$ and measurement noise $v_{k}^{i}$ are correlated; meanwhile, the measurement noises $v_{k}^{i}$ of the $i$ th subsystem and $v_{k}^{j}$ of the $j$ th subsystem are cross-correlated. The statistical properties satisfy as following:

$$
\begin{aligned}
& E\left(w_{k}\right)=0, \\
& E\left(v_{k}^{i}\right)=0, \\
& E\left(\left(\begin{array}{c}
w_{k} \\
v_{k}^{i}
\end{array}\right)\left(\begin{array}{ll}
w_{l}^{T} & \left(v_{k}^{j}\right)^{T}
\end{array}\right)\right)=\left(\begin{array}{cc}
Q_{k} \delta_{k, l} & S_{k}^{j} \delta_{k, l} \\
\left(S_{k}^{i}\right)^{T} \delta_{k, l} & R_{k}^{i, j} \delta_{k, l}
\end{array}\right),
\end{aligned}
$$

where $Q_{k}=Q_{k}{ }^{T}, R_{k}^{i, j}=\left(R_{k}^{j, i}\right)^{T}$, and $S_{k}^{i, j}=\left(S_{k}^{j, i}\right)^{T}$.

For the stored measurement sequence with random transmission delays, packet dropouts and packet disorders, the issue of distributed estimation is translated into finding the optimal state estimation $\widehat{x}_{L Z}(k \mid k)$ using logic $\mathrm{ZOH}$, which are fused and compensated by each local estimation $\widehat{x}_{L Z}^{i}\left(k-\tau^{i}\left(k_{1}\right) \mid k-\tau^{i}\left(k_{1}\right)\right)$.

\section{Estimation for Event-Triggered Signal Selection Method}

For the considered uncertain system (1)-(2), a distributed fusion estimation based on robust finite horizon filtering approach is investigated. Firstly, the augmented state vectors are presented to determine the upper bounds of the filtering and the prediction covariance matrices. Secondly, a linear delay compensation scheme based on estimation is proposed for dealing with the random transmission delays, which improves the computational efficiency. Thirdly, the packet dropouts are compensated by introducing one-step prediction estimation approach. At last, a weighted fusion estimation is presented to improve the estimation accuracy for the distributed system.

3.1. Augmented State Vector for Subsystem. For the $i$-th subsystem, suppose the current sampling time instant is $k$, and the stored data packet is $y_{L Z}^{i}(k)$. According to the role of logic $\mathrm{ZOH}$, the received valid data packet is $z^{i}\left(k-\tau^{i}\left(k_{1}\right)\right)$ with data delay $\tau^{i}\left(k_{1}\right)$. Owing to $\tau^{i}\left(k_{1}\right)=\tau^{i}\left(k_{2}\right)-\beta^{i}(k)$ and $\beta^{i}(k)>0$, define $t=k-\tau^{i}\left(k_{1}\right)$, and the measurement is reorganized from (2) and (5):

$$
y_{L Z}^{i}(k)=z^{i}(t)=\left(C_{t}^{i}+\mathscr{H}_{t}^{i} F_{t} E_{t}^{i}\right) x(t)+v_{t}^{i}
$$

Assume that the estimator has enough processing capability to calculate the optimal state estimation $\hat{x}_{L Z}^{i}(t \mid t)$ depending on the stored data $\left\{y_{L Z}^{i}(0), \cdots, y_{L Z}^{i}(k-1), y_{L Z}^{i}(k)\right\}$.

The objective of the robust finite horizon Kalman-type filtering for each subsystem is to obtain an optimal state estimation $\widehat{x}_{L Z}^{i}(k \mid k)$, which is derived from the guaranteed 
upper bound of estimation error covariance matrices. Applying the projection formula, the state estimation with random transmission delays is designed by the following recursive method based on the reorganized data packets:

$$
\begin{aligned}
\hat{x}_{L Z}^{i}(t \mid t)= & \widehat{x}_{L Z}^{i}(t \mid t-1) \\
& +K_{L Z, t}^{i}\left(z^{i}(t)-\widehat{C}_{L Z, t}^{i} \widehat{x}_{L Z}^{i}(t \mid t-1)\right), \\
\widehat{x}_{L Z}^{i}(t+1 \mid t)= & \widehat{A}_{L Z, t}^{i} \widehat{x}_{L Z}^{i}(t \mid t-1) \\
& +L_{L Z, t}^{i}\left(z^{i}(t)-\widehat{C}_{L Z, t}^{i} \widehat{x}_{L Z}^{i}(t \mid t-1)\right),
\end{aligned}
$$

where $\hat{x}_{L Z}^{i}(t \mid t)$ denotes the filter and $\widehat{x}_{L Z}^{i}(t+1 \mid t)$ is the predictor of the state $x(t)$ at time instant $t$ before being transmitted. Define $\tilde{e}_{L Z}^{i}(t) \triangleq x(t)-\widehat{x}_{L Z}^{i}(t \mid t-1)$ and $e_{L Z}^{i}(t) \triangleq x(t)-\hat{x}_{L Z}^{i}(t \mid t)$. Note that the prediction error and filtering error are forced to be smaller than the positivedefinite matrices $\bar{\Theta}_{L Z}^{i}(t)$ and $\bar{\Sigma}_{L Z}^{i}(t)$. For all uncertainties, the upper bounds are represented as following:

$$
\begin{aligned}
& E\left(\left(x(t)-\hat{x}_{L Z}^{i}(t \mid t)\right)\left(x(t)-\hat{x}_{L Z}^{i}(t \mid t)\right)^{T}\right) \\
& \quad \leq \bar{\Theta}_{L Z}^{i}(t) \\
& E\left(\left(x(t)-\hat{x}_{L Z}^{i}(t \mid t-1)\right)\left(x(t)-\hat{x}_{L Z}^{i}(t \mid t-1)\right)^{T}\right) \\
& \quad \leq \bar{\Sigma}_{L Z}^{i}(t) .
\end{aligned}
$$

Set $\widehat{C}_{L Z, t}^{i}, K_{L Z, t}^{i}, \widehat{A}_{L Z, t}^{i}$, and $L_{L Z, t}^{i}$ be filter parameters. Inspired by the adaptive gain [42], filter parameters are time-varying matrix which is online updated. It is worth noting that the system noise and measurement noise are different and the corresponding state-space model can be transformed the form as in (1) and (7) by using the augmentation method [26]. To solve the upper bounds from the filtering and prediction covariance matrices, the augmented state vectors are defined as follows:

$$
\begin{aligned}
& \widetilde{\Psi}_{L Z}^{i}(t)=\left[\begin{array}{c}
\tilde{e}_{L Z}^{i}(t) \\
\hat{x}_{L Z}^{i}(t \mid t-1)
\end{array}\right], \\
& \Psi_{L Z}^{i}(t)=\left[\begin{array}{c}
e_{L Z}^{i}(t) \\
\hat{x}_{L Z}^{i}(t \mid t)
\end{array}\right] .
\end{aligned}
$$

The reorganized measurement from (7) contains stochastic terms due to delayed sensor measurement. Therefore, it is necessary to derive the estimation error and obtain a corresponding upper bound. And then, an augmented state-space model combining system (1) and (8)-(12) are represented as

$$
\begin{aligned}
\Psi_{L Z}^{i}(t)= & \left(A_{L Z, t 1}^{i}+H_{L Z, t 1}^{i} F_{t} E_{L Z, t 1}^{\mathrm{i}}\right) \widetilde{\Psi}_{L Z}^{i}(t) \\
& +D_{L Z, t 1}^{i} v_{t}^{i}, \\
\widetilde{\Psi}_{L Z}^{i}(t+1)= & \left(A_{L Z, t 2}^{i}+H_{L Z, t 2}^{i} F_{t} E_{L Z, t 2}^{i}\right) \widetilde{\Psi}_{L Z}^{i}(t) \\
& +B_{L Z, t 2} w_{t}+D_{L Z, t 2}^{i} v_{t}^{i},
\end{aligned}
$$

with

$$
\begin{aligned}
& A_{L Z, t 1}^{i}=\left[\begin{array}{cc}
I-K_{L Z, t}^{i} C_{t}^{i} & K_{L Z, t}^{i}\left(\widehat{C}_{L Z, t}^{i}-C_{t}^{i}\right) \\
K_{L Z, t}^{i} C_{t}^{i} & I+K_{L Z, t}^{i}\left(C_{t}^{i}-\widehat{C}_{L Z, t}^{i}\right)
\end{array}\right], \\
& H_{L Z, t 1}^{i}=\left[\begin{array}{c}
-K_{L Z, t}^{i} \mathscr{H}_{t}^{i} \\
K_{L Z, t}^{i} \mathscr{H}_{t}^{i}
\end{array}\right] \text {, } \\
& E_{L Z, t 1}^{i}=\left[\begin{array}{ll}
E_{t}^{i} & E_{t}^{i}
\end{array}\right] \text {, } \\
& D_{L Z, t 1}^{i}=\left[\begin{array}{c}
-K_{L Z, t}^{i} \\
K_{L Z, t}^{i}
\end{array}\right], \\
& A_{L Z, t 2}^{i} \\
& =\left[\begin{array}{cc}
A_{t}-L_{L Z, t}^{i} C_{t}^{i} & A_{t}-\widehat{A}_{L Z, t}^{i}+L_{L Z, t}^{i}\left(\widehat{C}_{L Z, t}^{i}-C_{t}^{i}\right) \\
L_{L Z, t}^{i} C_{t}^{i} & \widehat{A}_{L Z, t}^{i}+L_{L Z, t}^{i}\left(C_{t}^{i}-\widehat{C}_{L Z, t}^{i}\right)
\end{array}\right], \\
& H_{L Z, t 2}^{i}=\left[\begin{array}{c}
\mathscr{F}_{t}^{i}-L_{L Z, t}^{i} \mathscr{H}_{t}^{i} \\
L_{L Z, t}^{i} \mathscr{H}_{t}^{i}
\end{array}\right] \text {, } \\
& E_{L Z, t 2}^{i}=\left[\begin{array}{ll}
E_{t}^{i} & E_{t}^{i}
\end{array}\right] \text {, } \\
& B_{L Z, t 2}=\left[\begin{array}{c}
B_{t} \\
0
\end{array}\right] \text {, } \\
& D_{L Z, t 2}^{i}=\left[\begin{array}{c}
-L_{L Z, t}^{i} \\
L_{L Z, t}^{i}
\end{array}\right] .
\end{aligned}
$$

Note that the augmented system (13)-(14) is a stochastic parameter system [43], and set the covariance matrices to be $\widetilde{\Sigma}_{L Z}^{i}(t)=E\left(\widetilde{\Psi}_{L Z}^{i}(t)\left(\widetilde{\Psi}_{L Z}^{i}(t)\right)^{T}\right)$ and $\widetilde{\Theta}_{L Z}^{i}(t)=$ $E\left(\Psi_{L Z}^{i}(t)\left(\Psi_{L Z}^{i}(t)\right)^{T}\right)$. Under (13)-(15), the Riccati-like equations for estimation error covariances are evolved as follows:

$$
\begin{aligned}
\widetilde{\Theta}_{L Z}^{i}(t)= & \left(A_{L Z, t 1}^{i}+H_{L Z, t 1}^{i} F_{t} E_{L Z, t 1}^{i}\right) \widetilde{\Sigma}_{L Z}^{i}(t) \\
& \cdot\left(A_{L Z, t 1}^{i}+H_{L Z, t 1}^{i} F_{t} E_{L Z, t 1}^{i}\right)^{T} \\
& +D_{L Z, t 1}^{i} R_{t}^{i}\left(D_{L Z, t 1}^{i}\right)^{T},
\end{aligned}
$$

and

$$
\begin{aligned}
\widetilde{\Sigma}_{L Z}^{i}(t+1)= & \left(A_{L Z, t 2}^{i}+H_{L Z, t 2}^{i} F_{t} E_{L Z, t 2}^{i}\right) \widetilde{\Sigma}_{L Z}^{i}(t) \\
& \cdot\left(A_{L Z, t 2}^{i}+H_{L Z, t 2}^{i} F_{t} E_{L Z, t 2}^{i}\right)^{T} \\
& +B_{L Z, t 2} Q_{t} B_{L Z, t 2}^{T}+D_{L Z, t 2}^{i} R_{t}^{i}\left(D_{L Z, t 2}^{i}\right)^{T} \\
& +B_{L Z, t 2} S_{t}^{i}\left(D_{L Z, t 2}^{i}\right)^{T}+D_{L Z, t 2}^{i}\left(S_{t}^{i}\right)^{T} \\
& \cdot B_{L Z, t 2}^{T} .
\end{aligned}
$$

It is noted that the deterministic uncertainty $F_{t}$ appears in (16) and (17). Therefore, it is impossible to have the exact value 
of the covariance matrices $\widetilde{\Theta}_{L Z}^{i}(t)$ and $\widetilde{\Theta}_{L Z}^{i}(t)$. An alternative approach is to find a set of upper bounds and then obtain the minimum with respect to filter parameters.

3.2. Upper Bound for Estimation Covariance. Based on the augmented state vectors, the corresponding filtering error covariance and prediction error covariance are calculated. The objective of designing the robust finite horizon Kalmantype filter needs to probe the appropriate filter parameters from estimation error covariance matrices.

To obtain a guaranteed upper bound for minimizing the estimation error covariance, the following lemmas are introduced.

Lemma 3. Assume that matrices $A, H, E$, and $F$ have compatible dimensions such that $F F^{T} \leq I$. The inequality $\alpha^{-1} I-E X E^{T}>0$ lets $X$ be a symmetric positive definite matrix and lets $\alpha>0$ be an arbitrary positive constant. Then, the following inequality holds:

$$
\begin{aligned}
& (A+H F E) X(A+H F E)^{T} \\
& \quad \leq A\left(X^{-1}-\alpha E^{T} E\right)^{-1} A^{T}+\alpha^{-1} H H^{T},
\end{aligned}
$$

and $\left(X^{-1}-\alpha E^{T} E\right)^{-1}=X+X E^{T}\left(\alpha^{-1} I-E X E^{T}\right)^{-1} E X$ is obtained from the matrix inversion lemma $[24,43]$.

Lemma 4. When $0 \leq t \leq k$, supposing that $X$ and $Y$ are symmetric positive definite matrices, the functions meet the conditions $s_{t}(X)=s_{t}^{T}(X) \in \mathbb{R}^{n \times n}$ and $h_{t}(X)=h_{t}^{T}(X) \in \mathbb{R}^{n \times n}$. If there exists $Y>X$, such that $s_{t}(Y) \geq s_{t}(X)$ and $h_{t}(Y) \geq$ $s_{t}(Y)$, then the solutions $M_{t}$ and $N_{t}$ in terms of the recursive equations

$$
\begin{gathered}
M_{t+1}=s_{t}\left(M_{t}\right), \\
N_{t+1}=h_{t}\left(N_{t}\right), \\
M_{0}=N_{0}>0
\end{gathered}
$$

satisfy $M_{t} \leq N_{t}[24,43]$.

Theorem 5. For the formula $(A+H F E) X(A+H F E)^{T}$ from (16) and (17) according to Lemmas 3 and 4, if there exists a positive scalar $\alpha_{t}$ and a symmetric positive definite matrix $\Sigma_{L Z}^{i}(t)$ satisfying $\alpha_{t}^{-1} I-E_{L Z, t 2}^{i} \Sigma_{L Z}^{i}(t)\left(E_{L Z, t 2}^{i}\right)^{T}>0$, then $\widetilde{\Sigma}_{L Z}^{i}(t) \leq \Sigma_{L Z}^{i}(t)$ and $\widetilde{\Theta}_{L Z}^{i}(t) \leq \Theta_{L Z}^{i}(t)$, where $\widetilde{\Sigma}_{L Z}^{i}(t)$ and $\widetilde{\Theta}_{L Z}^{i}(t)$ are evolved from (16) and (17), respectively. Therefore, the upper bounds $\Theta_{L Z}^{i}(t)$ and $\Sigma_{L Z}^{i}(t+1)$ are computed by the following recursive equations:

$$
\begin{aligned}
\Theta_{L Z}^{i}(t)= & A_{L Z, t 1}^{i} \Sigma_{L Z}^{i}(t)\left(A_{L Z, t 1}^{i}\right)^{T} \\
& +\alpha_{t}^{-1} H_{L Z, t 1}^{i}\left(H_{L Z, t 1}^{i}\right)^{T} \\
& +D_{L Z, t 1}^{i} R_{t}^{i}\left(D_{L Z, t 1}^{i}\right)^{T}+A_{L Z, t 1}^{i} \Sigma_{L Z}^{i}(t) \\
& \cdot\left(E_{L Z, t 1}^{i}\right)^{T}
\end{aligned}
$$

$$
\begin{gathered}
\times\left(\alpha_{t}^{-1} I-E_{L Z, t 1}^{i} \Sigma_{L Z}^{i}(t)\left(E_{L Z, t 1}^{i}\right)^{T}\right)^{-1} \\
\cdot E_{L Z, t 1}^{i} \Sigma_{L Z}^{i}(t)\left(A_{L Z, t 1}^{i}\right)^{T}
\end{gathered}
$$

and

$$
\begin{aligned}
& \Sigma_{L Z}^{i}(t+1)=A_{L Z, t 2}^{i} \Sigma_{L Z}^{i}(t)\left(A_{L Z, t 2}^{i}\right)^{T} \\
& +\alpha_{t}^{-1} H_{L Z, t 2}^{i}\left(H_{L Z, t 2}^{i}\right)^{T}+A_{L Z, t 2}^{i} \Sigma_{L Z}^{i}(t)\left(E_{L Z, t 2}^{i}\right)^{T} \\
& \cdot\left(\alpha_{t}^{-1} I-E_{L Z, t 2}^{i} \Sigma_{L Z}^{\mathrm{i}}(t)\left(E_{L Z, t 2}^{i}\right)^{T}\right)^{-1} \\
& \quad \times E_{L Z, t 2}^{i} \Sigma_{L Z}^{i}(t)\left(A_{L Z, t 2}^{i}\right)^{T}+B_{L Z, t 2}^{i} Q_{t}^{i}\left(B_{L Z, t 2}^{i}\right)^{T} \\
& +D_{L Z, t 2}^{i} R_{t}^{i}\left(D_{L Z, t 2}^{i}\right)^{T}+B_{L Z, t 2} S_{t}^{i}\left(D_{L Z, t 2}^{i}\right)^{T} \\
& +D_{L Z, t 2}^{i}\left(S_{t}^{i}\right)^{T} B_{L Z, t 2}^{T} .
\end{aligned}
$$

Proof. The proof is derived from Lemmas 3 and 4. Meanwhile, the similar derivation process is referred in [24, 43].

Based on Theorem 5 and Kalman-type filtering, referring to (17), suppose that the error covariance matrix is denoted as the following form:

$$
\Sigma_{L Z}^{i}(t)=\left[\begin{array}{cc}
\bar{\Sigma}_{L Z}^{i}(t) & 0 \\
0 & P(t)-\bar{\Sigma}_{L Z}^{i}(t)
\end{array}\right],
$$

where $\bar{\Sigma}_{L Z}^{i}(t)=E\left(\tilde{e}_{L Z}^{i}(t)\left(\tilde{e}_{L Z}^{i}(t)^{T}\right)\right)$ and $P(t)=E\left(x(t) x^{T}(t)\right)$.

Then, the upper bound of error covariance matrix is defined as follows:

$$
\begin{gathered}
E\left(e_{L Z}^{i}(t)\left(e_{L Z}^{i}(t)\right)^{T}\right)=\left[\begin{array}{ll}
I & 0
\end{array}\right] \widetilde{\Theta}_{L Z}^{i}(t)\left[\begin{array}{l}
I \\
0
\end{array}\right] \\
\leq\left[\begin{array}{ll}
I & 0
\end{array}\right] \Theta_{L Z}^{i}(t)\left[\begin{array}{l}
I \\
0
\end{array}\right]=\bar{\Theta}_{L Z}^{i}(t)
\end{gathered}
$$

and

$$
\begin{aligned}
E & \left(\tilde{e}_{L Z}^{i}(t+1)\left(\tilde{e}_{L Z}^{i}(t+1)\right)^{T}\right) \\
& =\left[\begin{array}{ll}
I & 0
\end{array}\right] \widetilde{\Sigma}_{L Z}^{i}(t+1)\left[\begin{array}{l}
I \\
0
\end{array}\right] \leq\left[\begin{array}{ll}
I & 0
\end{array}\right] \Sigma_{L Z}^{i}(t+1)\left[\begin{array}{l}
I \\
0
\end{array}\right] \\
& =\bar{\Sigma}_{L Z}^{i}(t+1) .
\end{aligned}
$$

In order to obtain $\bar{\Sigma}_{L Z}^{i}(t)$ and $P(t)$, the optimal values of the proposed robust finite horizon Kalman-type filtering in (8) and (9) are derived from the following Theorem 6.

Theorem 6. At the current time instant $k$, the received valid measurement $y_{L Z}^{i}(k)$ contains the received transmission delay 
$\tau^{i}\left(k_{1}\right)$. Set $t=k-\tau^{i}\left(k_{1}\right)$, and $\alpha_{t}$ be a positive scalar. $\bar{\Sigma}_{L Z}^{i}(t)$ and $P(t)$ are the positive definite solutions for the following discretetime Riccati-like iterations:

$$
\begin{aligned}
\bar{\Theta}_{L Z}^{i}(t) & \\
= & \bar{\Sigma}_{L Z}^{i}(t)+\bar{\Sigma}_{L Z}^{i}(t)\left(E_{t}^{i}\right)^{T}\left(\widetilde{M}_{L Z, t}^{i}\right)^{-1} E_{t}^{i} \bar{\Sigma}_{L Z}^{i}(t) \\
& -\Lambda_{L Z}^{i}(t)\left(\Xi_{L Z}^{i}(t)\right)^{-1}\left(\Lambda_{L Z}^{i}(t)\right)^{T}, \\
\bar{\Sigma}_{L Z}^{i}(t+1) & \\
= & A_{t} \bar{\Sigma}_{L Z}^{i}(t)\left(I+\left(E_{t}^{i}\right)^{T}\left(M_{L Z, t}^{i}\right)^{-1} E_{t}^{i} \bar{\Sigma}_{L Z}^{i}(t)\right) A_{t}^{T} \\
& -\Delta_{L Z}^{i}(t)\left(\Xi_{L Z}^{i}(t)\right)^{-1}\left(\Delta_{L Z}^{i}(t)\right)^{T}+B_{t} Q_{t} B_{t}^{T} \\
& +\alpha_{t}^{-1} \mathscr{F}_{t} \mathscr{F}_{t}^{T}, \\
P(t+ & 1) \\
= & A_{t}\left(P^{-1}(t)-\alpha_{t}\left(E_{t}^{i}\right)^{T} E_{t}^{i}\right)^{-1} A_{t}^{T}+\alpha_{t}^{-1} \mathscr{F}_{t} \mathscr{F}_{t}^{T} \\
& +B_{t} Q_{t} B_{t}^{T},
\end{aligned}
$$

where $\Lambda_{L Z}^{i}(t)=\bar{\Sigma}_{L Z}^{i}(t)\left(C_{t}^{i}\right)^{T}+\bar{\Sigma}_{L Z}^{i}(t)\left(E_{t}^{i}\right)^{T}\left(M_{L Z, t}^{i}\right)^{-1} E_{t}^{i} \bar{\Sigma}_{L Z}^{i}(t)\left(C_{t}^{i}\right)^{T}$ and $\Delta_{L Z}^{i}(t)=A_{t} \bar{\Sigma}_{L Z}^{i}(t)\left(I+\left(E_{t}^{i}\right)^{T}\left(M_{L Z, t}^{i}\right)^{-1} E_{t}^{i} \bar{\Sigma}_{L Z}^{i}(t)\right)\left(C_{t}^{i}\right)^{T}+$ $\alpha_{t}^{-1} \mathscr{F}_{t}\left(\mathscr{H}_{t}^{i}\right)^{T}+B_{t} S_{t}^{i}$. They satisfy $P^{-1}(t)-\alpha_{t}\left(E_{t}^{i}\right)^{T} E_{t}^{i}>0$ and $M_{L Z, t}^{i}=\alpha_{t}^{-1} I-E_{t}^{i} \bar{\Sigma}_{L Z}^{i}(t)\left(E_{t}^{i}\right)^{T}>0$, respectively.

Then, the robust Kalman-type filtering given in (8)-(9) is designed by the filter parameters:

$$
\begin{aligned}
& \widehat{C}_{L Z, t}^{i}=C_{t}^{i}\left(I+\bar{\Sigma}_{L Z}^{i}(t)\left(E_{t}^{i}\right)^{T}\left(M_{L Z, t}^{i}\right)^{-1} E_{t}^{i}\right), \\
& K_{L Z, t}^{i}=\Lambda_{L Z}^{i}(t)\left(\Xi_{L Z}^{i}(t)\right)^{-1}, \\
& \widehat{A}_{L Z, t}^{i}=A_{t}\left(I+\bar{\Sigma}_{L Z}^{i}(t)\left(E_{t}^{i}\right)^{T}\left(M_{L Z, t}^{i}\right)^{-1} E_{t}^{i}\right), \\
& L_{L Z, t}^{i}=\Delta_{L Z}^{i}(t)\left(\Xi_{L Z}^{i}(t)\right)^{-1},
\end{aligned}
$$

in which $\Xi_{L Z}^{i}(t)=C_{t}^{i} \bar{\Sigma}_{L Z}^{i}(t)\left(I+\left(E_{t}^{i}\right)^{T}\left(M_{L Z, t}^{i}\right)^{-1} E_{t}^{i} \bar{\Sigma}_{L Z}^{i}(t)\right)\left(C_{t}^{i}\right)^{T}+$ $\alpha_{t}^{-1} \mathscr{H}_{t}^{i}\left(\mathscr{H}_{t}^{i}\right)^{T}+R_{t}^{i}$ and $\widetilde{M}_{L Z, t}^{i}=\alpha_{t}^{-1} I-E_{t}^{i} P(t)\left(E_{t}^{i}\right)^{T}$.

Proof. This theorem follows from the upper bounds of minimizing estimation error covariance matrices. The proof procedure is proved in Appendix A.

3.3. Linear Compensation for Delays. The obtained local optimal state estimation $\hat{x}_{L Z}^{i}(t \mid t)$ is used for compensating the estimated value $\hat{x}_{L Z}^{i}(k \mid t)$ at the current time instant $k$. Meanwhile, the proposed linear delay compensation method based on estimation is employed to reduce the computational burden.

In order to estimate the state $\hat{x}_{L Z}^{i}(k \mid t)$ depending on the largest delay $N$ and the current received signal with transmission delay $\tau\left(k_{1}\right)$, the predicted value $\hat{x}_{L Z}^{i}(t+1 \mid t)$ is used for the linear compensation method:

$$
\widehat{x}_{L Z}^{i}(k \mid t)=\left(1-\frac{\tau^{i}\left(k_{1}\right)-1}{N}\right) \widehat{x}_{L Z}^{i}(t+1 \mid t) .
$$

Remark 7 . When the estimator receives the valid signals at the time instant $k=t+\tau(k)$ with transmission delay $\tau(k)$, the sensor sends an acknowledgement (ACK) signal to the estimator. The ACK signal is assigned the highest transmission priority, and then the transmission delay is negligible [2]. Once the estimator receives the ACK signal, the estimated state is used by the measurement $z(t)$. Then, for the $i$ th sensor using logic $\mathrm{ZOH}$, take the criterion into account that the ACK data packet before being transmitted is allocated the highest transmission priority, and the received data delay would be ignored for this reason. Therefore, the filter $\hat{x}_{L Z}^{i}(t \mid t)$ is used for the state estimation $\hat{x}_{L Z}^{i}(k \mid t)$ given in (32).

Remark 8. The local state estimation $\hat{x}_{L Z}^{i}(k \mid t)$ is an approximate estimation with delay-free. Using the logic $\mathrm{ZOH}$, the estimated state for the most recent data packet $\hat{x}_{L Z}^{i}(t \mid t)$ is stored, and the estimated state $\hat{x}_{L Z}^{i}(k \mid k)$ can be computed. Otherwise, if there is no arrived signal from the $i$ th sensor at time instant $k$, the estimated state will be compensated by one-step prediction of $\left\{\hat{x}_{L Z}^{i}(k-1 \mid k-\right.$ 1), $\left.\cdots, \widehat{x}_{L Z}^{i}(k-\tau \mid k-\tau)\right\}$ [29]. Therefore, the estimation accuracy is less than the one-step predication compensation strategy. However, due to the growing error accumulation and computation burden using the one-step predication, the proposed linear delay compensation method is used to suppress the computational complexity and alleviate the negative effect of the transmission delays.

For the next sampling time $k+1$, the arrived data is $y_{L Z}^{i}(k+$ 1 ) with delay $\tau^{i}\left(k_{1}+1\right)$; meanwhile, the received measured output is $z^{i}(s)$ with time-stamp before being transmitted, which satisfies $s=k+1-\tau^{i}\left(k_{1}+1\right)$. Since the disordered packets are discarded using the logic $\mathrm{ZOH}, s \geq t$ is satisfied. Therefore, two cases for calculating the filter $\hat{x}_{L Z}^{i}(s \mid s)$ is shown as follows.

Case 1. If $s=t$ or $s=t+1$, the state estimation $\widehat{x}_{L Z}^{i}(s \mid s)$ is derived from (8), and the filter parameters are calculated by the iterative equations based on Theorem 6 .

Case 2. If $s>t+1$, the estimated state $\hat{x}_{L Z}^{i}(s \mid s)$ will be compensated by one-step prediction $\widehat{x}_{L Z}^{i}(t+1 \mid t+1)$ given in (8)-(9) with the artificial delay $\tau^{s t}(k)=s-t>1$ and the reorganized state estimation sequence is compensated by

$$
\left\{\hat{x}_{L Z}^{i}(t+1 \mid t+1), \cdots, \hat{x}_{L Z}^{i}\left(t+\tau^{s t}(k) \mid t+\tau^{s t}(k)\right)\right\} .
$$

Under the given systems (1) and (2), the compensated estimation error covariance matrices and filter parameters are computed by the recursion formulas in (25)-(31). 
Remark 9. It is worth noting that the artificial delay is determined by the current received data packet and the previous received data packet with time-stamp. For the random transmission delays, an optimal artificial delay is obtained from the signal selection scheme of logic $\mathrm{ZOH}$. More importantly, due to the packet dropout phenomenon, the objective of one-step prediction with the artificial delay is used to establish the fusion estimation for each subsystem at the same sampling time.

3.4. Distributed Weighted Fusion Estimation. Based on Theorem 6, the local state estimation is obtained. For each subsystem, taking into account of the network-induced random transmission delays, packet dropouts, and out-oforder packets, the current estimated state is approximately calculated from (32). In order to improve the state estimation accuracy, the distributed fusion estimation is investigated. With the aid of the linear delay compensation strategy based on estimation and weighted fusion criterion, minimizing estimation error cross-covariance matrices are applied to perform information exchange between any two subsystems.

Theorem 10. For the linear discrete-time system (1) and (2), based on the linear delay compensation scheme, the upper bound of filtering error cross-covariance matrix $\bar{\Theta}_{L Z}^{i, j}(t)$ and prediction error cross-covariance matrix $\bar{\Sigma}_{L Z}^{i, j}(t+1)$ between $i$ th and $j$ th subsystems at time instant $k$ have the following expressions:

$$
\begin{aligned}
& \bar{\Theta}_{L Z}^{i, j}(t)=\left[\begin{array}{ll}
I & 0
\end{array}\right] \Theta_{L Z}^{i, j}(t)\left[\begin{array}{l}
I \\
0
\end{array}\right]=\left(I-K_{L Z, t}^{i} C_{t}^{i}\right) \bar{\Sigma}_{L Z}^{i, j}(t) \\
& \cdot\left(I-K_{L Z, t}^{j} C_{t}^{j}\right)^{T}+\left(K_{L Z, t}^{i}\left(\widehat{C}_{L Z, t}^{i}-C_{t}^{i}\right)\right)(P(t) \\
& \left.-\bar{\Sigma}_{L Z}^{i, j}(t)\right)\left(K_{L Z, t}^{j}\left(\widehat{C}_{L Z, t}^{j}-C_{t}^{j}\right)\right)^{T}+\left(\bar{\Sigma}_{L Z}^{i}(t)\right. \\
& \left.+K_{L Z, t}^{i}\left(\widehat{C}_{L Z, t}^{i}\left(P(t)-\bar{\Sigma}_{L Z}^{i}(t)\right)-C_{t}^{i} P(t)\right)\right)\left(E_{t}^{i}\right)^{T} \\
& +\left(\widetilde{M}_{L Z, t}^{i, j}\right)^{-1} E_{t}^{j} \times\left(\bar{\Sigma}_{L Z}^{j}(t)\right. \\
& \left.+K_{L Z, t}^{j}\left(\widehat{C}_{L Z, t}^{j}\left(P(t)-\bar{\Sigma}_{L Z}^{j}(t)\right)-C_{t}^{j} P(t)\right)\right)^{T} \\
& +\alpha_{t}^{-1} K_{L Z, t}^{i} \mathscr{H}_{t}^{i}\left(\mathscr{H}_{t}^{j}\right)^{T}\left(K_{L Z, t}^{j}\right)^{T} \\
& +K_{L Z, t}^{i} R_{t}^{i, j}\left(K_{L Z, t}^{j}\right)^{T}
\end{aligned}
$$

and

$$
\begin{aligned}
& \bar{\Sigma}_{L Z}^{i, j}(t+1)=\left[\begin{array}{ll}
I & 0
\end{array}\right] \Sigma_{L Z}^{i, j}(t+1)\left[\begin{array}{l}
I \\
0
\end{array}\right]=\left(A_{t}-L_{L Z, t}^{i} C_{t}^{i}\right) \\
& \cdot \bar{\Sigma}_{L Z}^{i, j}(t)\left(A_{t}-L_{L Z, t}^{j} C_{t}^{j}\right)^{T}+B_{t} Q_{t} B_{t}^{T} \\
& +L_{L Z, t}^{i} R_{t}^{i, j}\left(L_{L Z, t}^{j}\right)^{T}+\left(A_{t}-\widehat{A}_{L Z, t}^{i}\right. \\
& \left.+L_{L Z, t}^{i}\left(\widehat{C}_{L Z, t}^{i}-C_{t}^{i}\right)\right)\left(P(t)-\bar{\Sigma}_{L Z}^{i, j}(t)\right) \times\left(A_{t}\right.
\end{aligned}
$$

$$
\begin{aligned}
& \left.-\widehat{A}_{L Z, t}^{j}+L_{L Z, t}^{j}\left(\widehat{C}_{L Z, t}^{j}-C_{t}^{j}\right)\right)^{T}+\left(\left(A_{t}-L_{L Z, t}^{i} C_{t}^{i}\right)\right. \\
& \cdot \bar{\Sigma}_{L Z}^{i}(t)+\left(A_{t}-\widehat{A}_{L Z, t}^{i}+L_{L Z, t}^{i}\left(\widehat{C}_{L Z, t}^{i}-C_{t}^{i}\right)\right) \\
& \left.\times\left(P(t)-\bar{\Sigma}_{L Z}^{i}(t)\right)\right)\left(E_{t}^{i}\right)^{T}\left(\widetilde{M}_{L Z, t}^{i, j}\right)^{-1} E_{t}^{j}\left(\bar{\Sigma}_{L Z}^{j}(t)\right. \\
& \cdot\left(A_{t}-L_{L Z, t}^{j} C_{t}^{j}\right)^{T}+\left(P(t)-\bar{\Sigma}_{L Z}^{j}(t)\right) \\
& \left.\cdot\left(A_{t}-\widehat{A}_{L Z, t}^{j}+L_{L Z, t}^{j}\left(\widehat{C}_{L Z, t}^{j}-C_{t}^{j}\right)\right)^{T}\right)+\alpha_{t}^{-1}\left(\mathscr{F}_{t}\right. \\
& \left.-L_{L Z, t}^{i} \mathscr{H}_{t}^{i}\right)\left(\mathscr{F}_{t}-L_{L Z, t}^{j} \mathscr{H}_{t}^{j}\right)^{T}-B_{t} S_{t}^{j}\left(L_{L Z, t}^{j}\right)^{T} \\
& -L_{L Z, t}^{i}\left(S_{t}^{i}\right)^{T} B_{t}^{T},
\end{aligned}
$$

where $\widetilde{M}_{L Z, t}^{i, j}=\alpha_{t}^{-1} I-E_{t}^{i} P(t)\left(E_{t}^{j}\right)^{T}$ and $M_{L Z, t}^{i, j}=\alpha_{t}^{-1} I-$ $E_{t}^{i} \bar{\Sigma}_{L Z}^{i, j}(t)\left(E_{t}^{j}\right)^{T}$.

Without loss of generality, according to the estimated state $\widehat{x}_{L Z}^{i}(k \mid t)$ and $\widehat{x}_{L Z}^{j}(k \mid t)$ of the ith and $j$-th subsystems, respectively, the upper bound of estimation error cross-covariance matrix $\Pi_{L Z}^{i, j}(k) \triangleq E\left[\tilde{x}_{L Z}^{i}(k \mid t)\left(\tilde{x}_{L Z}^{j}(k \mid t)\right)^{T}\right]$ is obtained as following:

$$
\begin{aligned}
& \Pi_{L Z}^{i, j}(k) \leq \bar{\Pi}_{L Z}^{i, j}(k) \\
& =\left(1-\frac{\tau^{i}\left(k_{1}\right)-1}{N}\right)\left(1-\frac{\tau^{j}\left(k_{1}\right)-1}{N}\right) \bar{\Sigma}_{L Z}^{i, j}(t+1) .
\end{aligned}
$$

Proof. The proof procedure is similar to the previous derivation of Theorem 6 for logic $\mathrm{ZOH}$ signal selection method.

A parallel-series hybrid meta-heuristic optimisation method is then proposed in [44], which combines a hybrid topology binary particle swarm optimization. Therefore, this paper employs the linear minimum variance [15], and the optimal weighted fusion estimation can be used to minimize the trace of the fusion estimation error cross-covariance. Let $\widehat{x}_{L Z}^{i}(k \mid k), i=1, \cdots, L$ represent the local estimation of an $r$-dimensional stochastic vector $x(k)$ with network-induced phenomena. The distributed fusion estimator is designed as $\widehat{x}_{L Z}(k \mid k)$ also an $r$-dimensional vector, which is given as follows:

$$
\widehat{x}_{L Z}(k \mid k)=\Omega_{k}^{1} \hat{x}_{L Z}^{1}(k \mid k)+\cdots+\Omega_{k}^{L} \widehat{x}_{L Z}^{L}(k \mid k) .
$$

Therefore, referring to the result of $[10,29]$, the distributed fusion estimation performance is optimal, if and only if the weighted matrix given in (37) is determined by

$$
\left[\Omega_{k}^{1}, \cdots, \Omega_{k}^{L}\right]=\left(I_{0}^{T} \Pi_{L Z}^{-1}(k) I_{0}\right)^{-1} I_{0}^{T} \Pi_{L Z}^{-1}(k),
$$

where $I_{0}=\underbrace{\left[I_{r}, \cdots, I_{r}\right]^{T}}_{L}$ is an $r L \times r$ matrix and $\sum_{i=1}^{L} \Omega_{k}^{i}=I_{r}$. Moreover, 


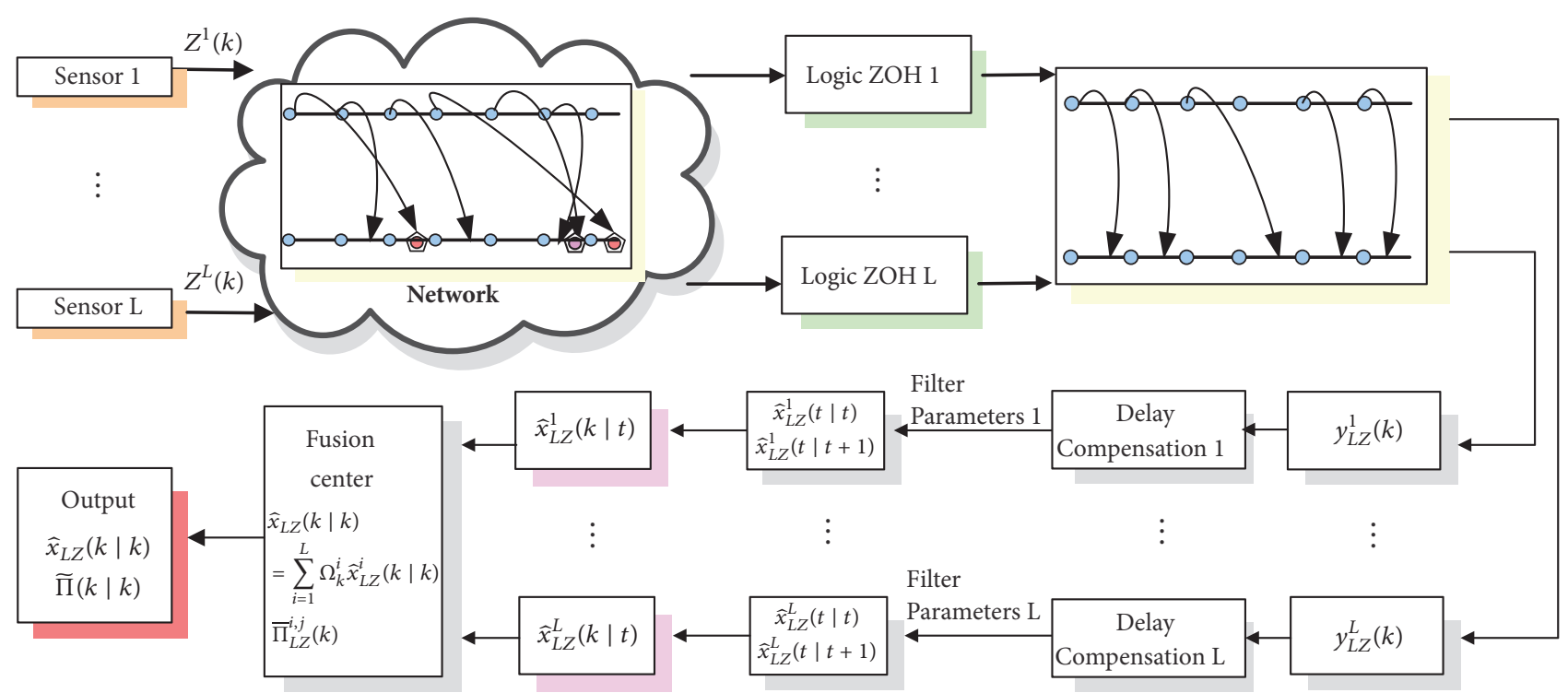

FIGURE 2: Computational procedure of distributed fusion estimation based on robust finite horizon filtering for logic $\mathrm{ZOH}$.

$$
\Pi_{L Z}(k)=\left[\begin{array}{ccc}
\Pi_{L Z}^{1,1}(k) & \cdots & \Pi_{L Z}^{1, L}(k) \\
& \vdots & \\
\Pi_{L Z}^{L, 1}(k) & \cdots & \Pi_{L Z}^{L, L}(k)
\end{array}\right]
$$

is a symmetrical positive definite matrix. The corresponding cross-covariance of the optimal information fusion estimator $\widetilde{\Pi}(k \mid k) \triangleq E\left[(x(k)-\widehat{x}(k \mid k))(x(k)-\widehat{x}(k \mid k))^{T}\right]$ is calculated as $\widetilde{\Pi}(k \mid k)=\left(I_{0}^{T} \Pi_{L Z}^{-1}(k) I_{0}\right)^{-1}$, which satisfies $\widetilde{\Pi}(k \mid k) \leq$ $\Pi_{L Z}^{i}(k) \leq \bar{\Pi}_{L Z}^{i}(k)$ if $i=j$; otherwise, $\widetilde{\Pi}(k \mid k) \leq \Pi_{L Z}^{i, j}(k) \leq$ $\bar{\Pi}_{L Z}^{i, j}(k)$.

The solution approach of distributed fusion estimation using robust finite horizon Kalman-type filtering is demonstrated in Figure 2.

Remark 11. The uncertain system introduces the logic $\mathrm{ZOH}$ signal selection method to deal with the network-induced transmission delays, packet dropouts, and out-of-order packets. Meanwhile, the packet disorders are dropped, and the received valid data are the latest data packet. As mentioned before, the linear compensation approach based on the estimation $\hat{x}_{L Z}^{i}(t \mid t)$ is used to obtain the local state, and the one-step prediction-based compensation approach is used to estimate the state $\widehat{x}_{L Z}^{i}\left(t+\tau^{s t}(k) \mid t+\tau^{s t}(k)\right)$, which is dependent on the artificial delay $\tau^{s t}(k)$ for the missing packets. On the other hand, to achieve information exchange, the distributed fusion estimation based on the weighted fusion criterion is obtained the consistent expression of state by the linear minimizing on variance matrix. As a theoretical basis, the fusion scheme possesses higher estimation accuracy than each local estimator.

\section{Numerical Simulation}

In this section, the results of a numerical example are demonstrated to illustrate the effectiveness of the proposed distributed fusion estimation strategy.

The considered target tracking systems with intermittent measurements are uncertain systems described in $[10,11,24$, 28]:

$$
\begin{aligned}
x(k+1)= & \left(\left[\begin{array}{ccc}
0.9 & T & \frac{T^{2}}{2} \\
0 & 0.9 & T \\
0 & 0 & 0.9
\end{array}\right]+\mathscr{F}_{k} F_{k} E_{k}\right) x(k) \\
& +\left[\begin{array}{c}
\frac{T^{2}}{2} \\
T \\
1
\end{array}\right] w_{k}, \quad k=1,2, \cdots \\
z^{i}(k)= & \left(C_{k}^{i}+\mathscr{H}_{k}^{i} F_{k} E_{k}^{i}\right) x(k)+v_{k}^{i}, \quad i=1,2,3 \\
w_{k}= & \eta_{k}, \\
v_{k}^{i}= & \zeta_{k}^{i} w_{k},
\end{aligned}
$$

where the sample period $T$ is set as $0.1 s$, the maximum of transmission delay is assumed to be $N=5$, and the timevarying parameter uncertainties satisfy $F_{k}=\sin (0.6 k)$. The state $x(k)=\left(\begin{array}{lll}s_{k} & \dot{s}_{k} & \ddot{s}_{k}\end{array}\right)^{T}$ denotes the position, velocity, and acceleration, respectively, of the target at time instant $k T$. Set matrices to be $\mathscr{F}_{k}=\left[\begin{array}{lll}0.1 & 0.1 & 0.1\end{array}\right]^{T}, E_{k}=\left[\begin{array}{lll}0.02 & 0.02 & 0.02\end{array}\right]$, $C_{k}^{1}=\left[\begin{array}{lll}0.6 & 0.8 & 1\end{array}\right], C_{k}^{2}=\left[\begin{array}{lll}1 & 0.8 & 0.5\end{array}\right], C_{k}^{3}=\left[\begin{array}{lll}0.3 & 1 & 0.7\end{array}\right]$, and $\mathscr{H}_{k}^{1}=\mathscr{H}_{k}^{2}=\mathscr{H}_{k}^{3}=0.8 . \eta_{k} \in \mathbb{R}$ is zero mean white noise with variance $\sigma_{\eta}^{2}=0.09$. Due to the cross-correlation between 


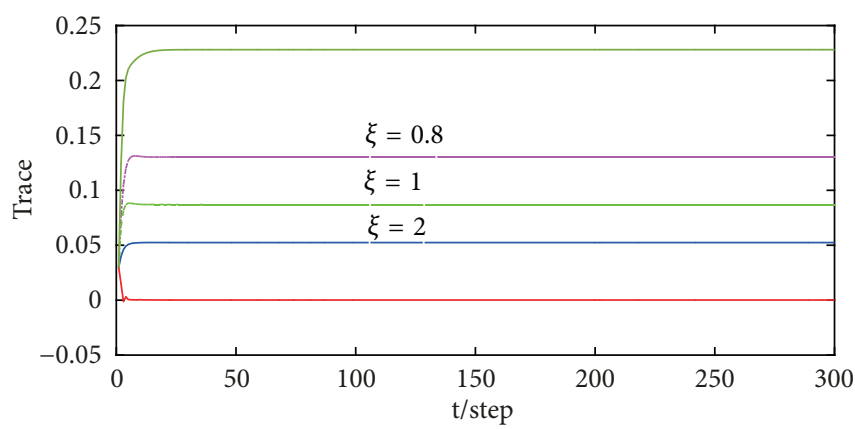

_ Trace of DFERFH by sensor 1

-.... Trace of DFERFH by sensor 2

.... Trace of DFERFH by sensor 3

— Trace of DFERFH by fusion estimation — Trace of IRFHKF

(a) Upper bounds for estimation error covariance matrices

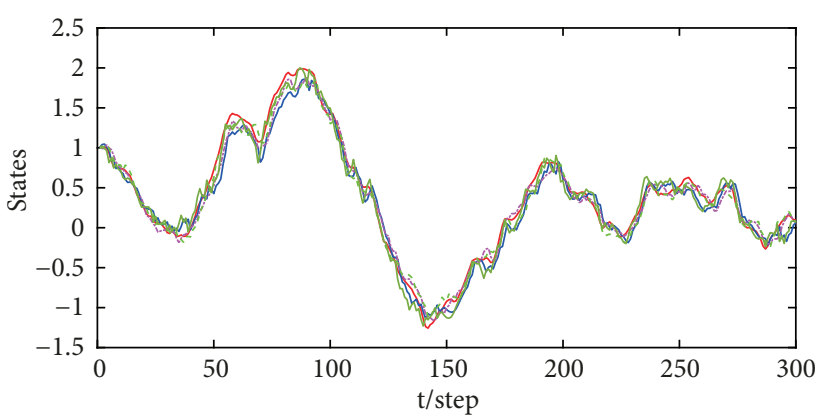

- Actual state of velocity

- Estimated state of DFERFH by sensor 1

-...- Estimated state of DFERFH by sensor 2

-... Estimated state of DFERFH by sensor 3

- Estimated state of IRFHKF

(c) Comparison of the estimated state for velocity

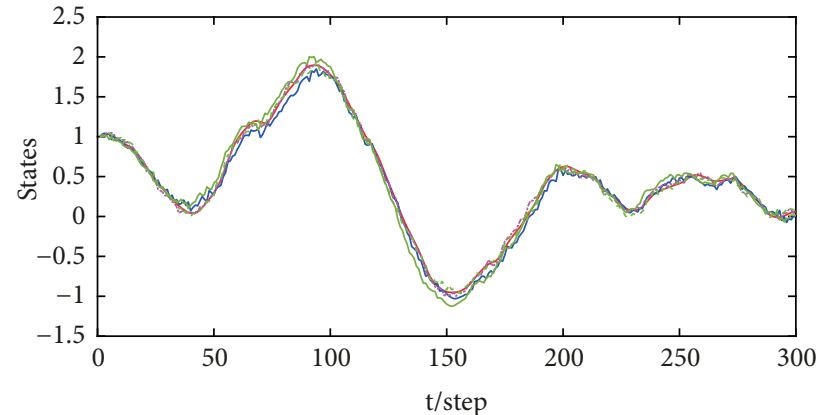

- Actual state of position

- Estimated state of DFERFH by sensor 1

-.-.-. Estimated state of DFERFH by sensor 2

.... Estimated state of DFERFH by sensor 3

— Estimated state of IRFHKF

(b) Comparison of the estimated state for position

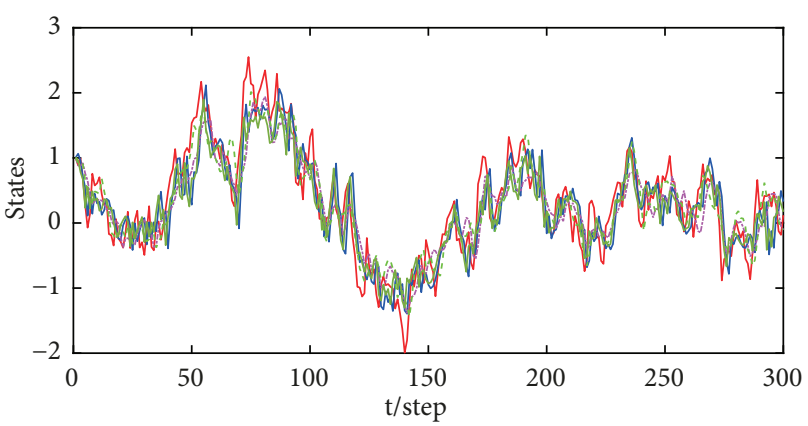

- Actual state of acceleration

- Estimated state of DFERFH by sensor 1

-..-... Estimated state of DFERFH by sensor 2

.... Estimated state of DFERFH by sensor 3

— Estimated state of IRFHKF

(d) Comparison of the estimated state for acceleration

FIgURE 3: Comparison of proposed method and IRFHKF.

process noise and measurement noise, the variable $\zeta_{k}^{i}$ in (43) determines the correlated strength and set $\zeta_{k}^{1}=2$ and $\zeta_{k}^{2}=$ $0.8, \zeta_{k}^{3}=1$, respectively. The process noise $w_{k}$ from (42) with unity variance $Q_{k}$, while, the covariance $R_{k}^{i, j}$ is denoted as $R_{k}^{i, j}=\zeta_{k}^{i} \zeta_{k}^{j} Q_{k}$ and the cross-covariance is $S_{k}^{i}=\zeta_{k}^{i} Q_{k}$ given in (43).

Without loss of generality, the initial values are set as $\alpha_{k}=3, \widehat{x}(0 \mid 0)=\mu_{0}=E(x(0))=\left[\begin{array}{lll}1 & 1 & 1\end{array}\right]^{T}$, and $P(0 \mid$ $0)=0.01 I_{3}$. The proposed method is verified from 300 time taken sampling points, and the results are obtained based on 100 Monte Carlo simulations. The comparison for the trace of estimation error covariance matrices is demonstrated in Figures $3(\mathrm{a})-3(\mathrm{~d})$. The estimated state $\widehat{x}(k \mid k)$ is verified by employing the improved robust finite-horizon Kalman filtering (IRFHKF) in [24], and the proposed distributed fusion estimation approach (DFERFH) with delay-free. As shown in Figure 3(a), the proposed estimator possesses less upper bound for the estimation error covariance matrices than IRFHKF method. It is worth noting that the fused upper bound of the estimation error cross-covariance matrix is minimum compared to other covariance matrices, since the weighted fusion criterion is again optimal in the minimum covariance sense. Comparing the estimation accuracy of the both methods from Figures 3(b)-3(d), the proposed method is suitable for designing the appropriate estimator to probe the optimal filter parameters. Taking the cross-correlation for noise into account, if the cross-correlation for noise is stronger, the dynamic tracking trajectory is closer the actual state to be estimated.

And then, the range of the performance indicator from minimum to maximum is presented in Table 1 . It implies that the upper bound of estimation error covariance matrices for the proposed method is less than the IRFHKF method. For the distributed networked system, the processing effectiveness of each subsystem is different, and it is determined by the system parameters and cross-correlated strength for noise. Note that the estimation performance is more accurate if the process and measurement noise have stronger crosscorrelation.

To further illustrate the effectiveness of the proposed method, the distributed estimation results of the estimated 
TABLE 1: Comparison of the filtering error covariance matrices.

\begin{tabular}{lcccc}
\hline Method & Position state & Velocity state & Acceleration state & Trace of covariance \\
\hline DFERFH 1 & $0.0096-0.0150$ & $0.0016-0.0100$ & $0.0100-0.0372$ & $0.0300-0.0533$ \\
DFERFH 2 & $0.0056-0.0100$ & $0.0033-0.0100$ & $0.0100-0.1258$ & $0.0291-0.1313$ \\
DFERFH 3 & $0.0084-0.0109$ & $0.0004-0.0100$ & $0.0100-0.0823$ & $0.0279-0.0883$ \\
IRFHKF & $0.0100-0.0267$ & $0.0100-0.0235$ & $0.0100-0.1779$ & $0.0300-0.2280$ \\
\hline
\end{tabular}
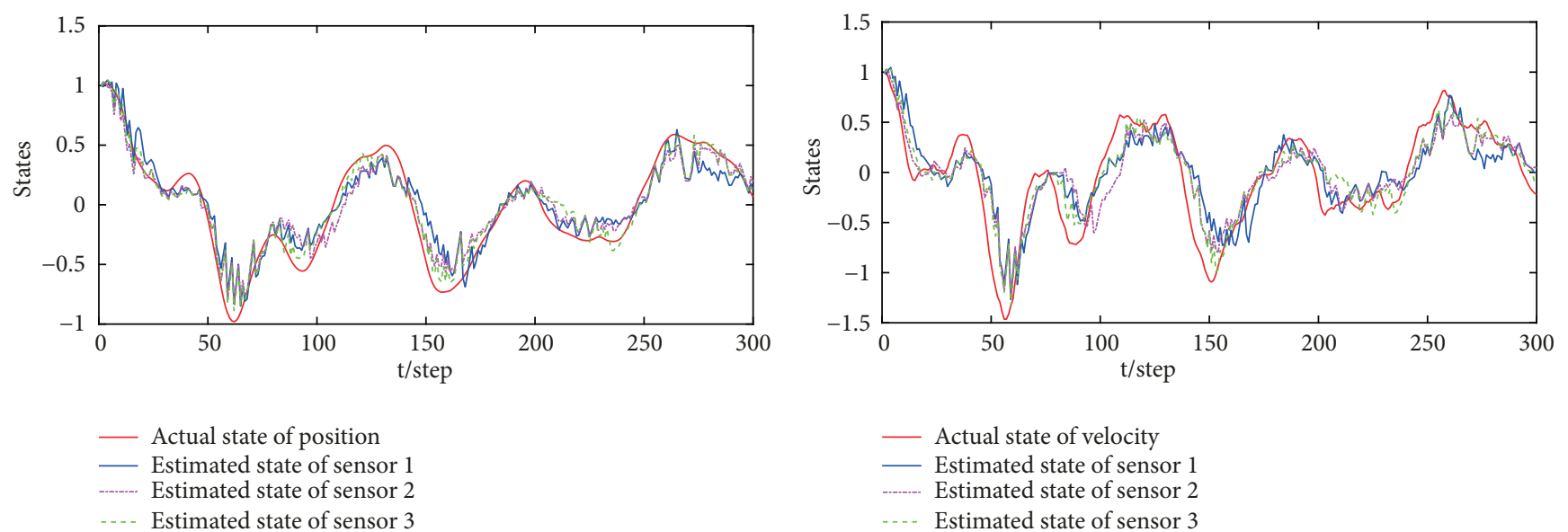

(a) Position and its estimated state

(b) Velocity and its estimated state

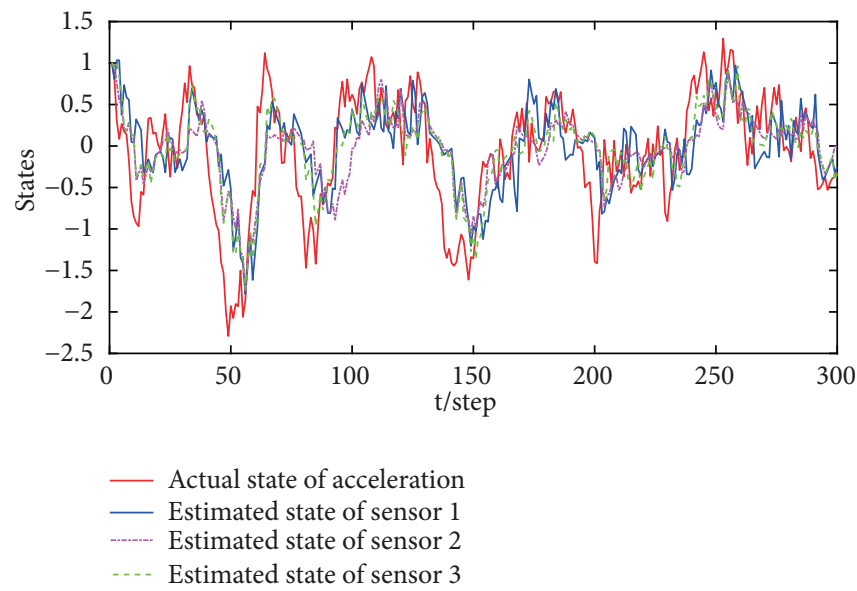

(c) Acceleration and its estimated state

FIGURE 4: Comparison of distributed estimation results using logic $\mathrm{ZOH}$.

states are shown in Figures 4(a)-4(c). The simulation results are obtained from the state estimation (8)-(9) by calculating the recursive equations in Theorems 6 and 10. Meanwhile, the robust finite horizon filtering and distributed weighted fusion estimation criteria are investigated for the logic $\mathrm{ZOH}$.

From Figures 4(a)-4(c), the logic ZOH scheme is able to discard out-of-order packets induced from random transmission delays and packet dropouts. Therefore, the distributed fusion estimation has the better performances for target tacking and computational efficiency with the linear delay compensation and one-step prediction estimation schemes.

In order to further analyze the performance of the proposed distributed fusion estimation approach with networkinduced phenomena, the mean square error (MSE) [10, 29,
45] values are displayed in Table 2. The comparison of the MSE values is composed of the states of position, velocity, and acceleration, as well as their estimated values for each subsystem, respectively.

Table 2 shows that based on robust finite horizon filtering of each subsystem, the proposed distributed fusion estimation is able to obtain the better estimation performance than each one.

The simulations are carried out, and the reordering error covariance criteria are used for comparing the dynamic tracking results. The comparison between the DFERFH and IRFHKF methods implies that the proposed estimation has better accuracy than IRFHKF method for the multi-step random delays, packet dropouts and out-of-order packets. 
TABLE 2: Comparison of mean square errors.

\begin{tabular}{lccc}
\hline Scheme & \multicolumn{2}{c}{$\begin{array}{c}\text { Mean of error covariance } \\
\text { velocity }\end{array}$} & 0.0330 \\
\hline Sensor 1 & 0.0042 & 0.0076 & 0.0299 \\
Sensor 2 & 0.0023 & 0.0065 & 0.0241 \\
Sensor 3 & 0.0016 & 0.0045 & 0.0006 \\
Fusion & 0.0005 & 0.0007 & 0.006 \\
\hline
\end{tabular}

Since the actual estimation error covariance is below the upper bound, for the actual estimated values, the proposed distributed fusion estimation method for network-induced phenomena has the better performance including close tracking of the system state and rapid convergence to a steady state.

\section{Conclusion}

This paper has investigated a class of uncertain systems with network-induced phenomena. To deal with packet dropouts and out-of-order packets generating from random transmission delays, the system model has been established by the event-triggered signal selection method of the logic $\mathrm{ZOH}$. With the aid of a linear delay compensation strategy, the optimal state estimation has been presented to overcome the influence of the limited communication capacity and suppress the computational burden. For the packet dropouts, the one-step prediction estimation method has been proposed to compensate the missing packets. The finite horizon filtering has been designed subsequently, so that the actual estimation error variance is less than the upper bound. Furthermore, the distributed estimation approach based on filtering has been introduced the weighted fusion criterion to obtain the consistent expression of the state. Thus, the fused estimation possesses higher accuracy than each local subsystem. As a theoretical result, the proposed modelling and distributed fusion estimation have better performance for dropping packet disorders. The numerical simulations for target tracking systems on three sensors have been performed to demonstrate the tracking performance and the effectiveness of the acquired theoretical results.

\section{Appendix}

\section{A. Proof of Theorem 6}

Since the solution of $\bar{\Theta}_{L Z}^{i}(t)$ and $\bar{\Sigma}_{L Z}^{i}(t+1)$ are derived from $\bar{\Sigma}_{L Z}^{i}(t)$ defined in (23) and (24), the upper bound $\Sigma_{L Z}^{i}(t)$ can be represented as follows $[23,24]$ :

$$
\begin{aligned}
\Sigma_{L Z}^{i}(t) & =\left[\begin{array}{cc}
\Sigma_{11}(t) & \Sigma_{12}(t) \\
\Sigma_{21}(t) & \Sigma_{22}(t)
\end{array}\right] \\
& =\left[\begin{array}{cc}
\bar{\Sigma}_{L Z}^{i}(t) & 0 \\
0 & P(t)-\bar{\Sigma}_{L Z}^{i}(t)
\end{array}\right] .
\end{aligned}
$$

Taking into account of the given recursive equations for $\bar{\Sigma}_{L Z}^{i}(t+1)$ and $P(t+1)$ in (26) and (27), respectively, as well as the estimation $\hat{x}_{L Z}^{i}(t \mid t)$ in (8), the estimated parameters $\widehat{C}_{L Z, t}^{i}$ and $K_{L Z, t}^{i}$ are determined by minimizing measurement and filtering error covariance matrices, respectively.

Step 1 (calculate filter parameter $\widehat{C}_{L Z, t}^{i}$ ). In the first place, set $t=k-\tau^{i}\left(k_{1}\right)$ and the measurement error $\tilde{y}_{L Z}^{i}(k)$ is defined as

$$
\begin{aligned}
\widetilde{y}_{L Z}^{i}(k) & =y_{L Z}^{i}(k)-\widehat{y}_{L Z}^{i}(k) \\
& =\left(A_{L Z, t_{3}}^{i}+H_{L Z, t 3}^{i} F_{t} E_{L Z, t 3}^{i}\right) \widetilde{\Psi}_{L Z}^{i}(t)+v_{t}^{i},
\end{aligned}
$$

where

$$
\begin{aligned}
A_{L Z, t 3}^{i} & =\left[\begin{array}{ll}
C_{t}^{i} & C_{t}^{i}-\widehat{C}_{L Z, t}^{i}
\end{array}\right], \\
H_{L Z, t 3}^{i} & =\mathscr{H}_{t}^{i}, \\
E_{L Z, t 3}^{\mathrm{i}} & =\left[\begin{array}{ll}
E_{t}^{i} & E_{t}^{i}
\end{array}\right] .
\end{aligned}
$$

Next, minimize the measurement error covariance matrix. Solve the upper bound of the measurement error covariance based on Lemmas 3 and 4 and (A.1):

$$
\begin{aligned}
& E\left(\tilde{y}_{L Z}^{i}(k)\left(\tilde{y}_{L Z}^{i}(k)\right)^{T}\right)=\left(A_{L Z, t 3}^{i}+H_{L Z, t 3}^{i} F_{t} E_{L Z, t 3}^{i}\right) \\
& \cdot \tilde{\Sigma}_{L Z}^{i}(t)\left(A_{L Z, t 3}^{i}+H_{L Z, t 3}^{i} F_{t} E_{L Z, t 3}^{i}\right)^{T}+R_{t}^{i} \\
& \quad \leq A_{L Z, t 3}^{i}\left(\Sigma_{L Z}^{i}(t)+\Sigma_{L Z}^{i}(t)\left(E_{L Z, t 3}^{i}\right)^{T}\right. \\
& \quad \times\left(\alpha_{t}^{-1} I-E_{L Z, t 3}^{i} \Sigma_{L Z}^{i}(t)\left(E_{L Z, t 3}^{i}\right)^{T}\right)^{-1} \\
& \left.\cdot E_{L Z, t 3}^{i} \Sigma_{L Z}^{i}(t)\right)\left(A_{L Z, t 3}^{i}\right)^{T}+\alpha_{t}^{-1} H_{L Z, t 3}^{i}\left(H_{L Z, t 3}^{i}\right)^{T} \\
& +R_{t}^{i}=\bar{\Pi}_{L Z}^{i}(t)=C_{t}^{i} \bar{\Sigma}_{L Z}^{i}(t)\left(C_{t}^{i}\right)^{T}+\left(C_{t}^{i}-\widehat{C}_{L Z, t}^{i}\right) \\
& +\left(P(t)-\bar{\Sigma}_{L Z}^{i}(t)\right)\left(C_{t}^{i}-\widehat{C}_{L Z, t}^{i}\right)^{T} \\
& +\alpha_{t}^{-1} \mathscr{H}_{t}^{i}\left(\mathscr{H}_{t}^{i}\right)^{T}+R_{t}^{i}+\left(C_{t}^{i} \bar{\Sigma}_{L Z}^{i}(t)\left(E_{t}^{i}\right)^{T}\right. \\
& \left.+\left(C_{t}^{i}-\widehat{C}_{L Z, t}^{i}\right)\left(P(t)-\bar{\Sigma}_{L Z}^{i}(t)\right)\left(E_{t}^{i}\right)^{T}\right) \times\left(\alpha_{t}^{-1} I\right. \\
& \left.+E_{t}^{i} P(t)\left(E_{t}^{i}\right)^{T}\right) \times\left(C_{t}^{i} \bar{\Sigma}_{L Z}^{i}(t)\left(E_{t}^{i}\right)^{T}\right. \\
& \left.\left.+\widehat{C}_{L Z, t}^{i}\right)\left(P(t)-\bar{\Sigma}_{L Z}^{i}(t)\right)\left(E_{t}^{i}\right)^{T}\right)^{T} \cdot
\end{aligned}
$$


Therefore, the first order derivative for $\widehat{C}_{L Z, t}^{i}$ is

$$
\begin{aligned}
& \frac{\partial \bar{\Pi}_{L Z}^{i}(t)}{\partial \widehat{C}_{L Z, t}^{i}}=-\left(C_{t}^{i}-\widehat{C}_{L Z, t}^{i}\right)\left(P(t)-\bar{\Sigma}_{L Z}^{i}(t)\right) \\
& \quad-C_{t}^{i} \bar{\Sigma}_{L Z}^{i}(t)\left(E_{t}^{i}\right)^{T}\left(\widetilde{M}_{L Z, t}^{i}\right)^{-1} E_{t}^{i}\left(P(t)-\bar{\Sigma}_{L Z}^{i}(t)\right) \\
& \quad-\left(C_{t}^{i}-\widehat{C}_{L Z, t}^{i}\right)\left(P(t)-\bar{\Sigma}_{L Z}^{i}(t)\right)\left(E_{t}^{i}\right)^{T}\left(\widetilde{M}_{L Z, t}^{i}\right)^{-1} \\
& \quad \cdot E_{t}^{i}\left(P(t)-\bar{\Sigma}_{L Z}^{i}(t)\right)=0 .
\end{aligned}
$$

And then, $\widehat{C}_{L Z, t}^{i}$ is derived by the straightforward transformation:

$$
\begin{aligned}
& \widehat{C}_{L Z, t}^{i}=C_{t}^{i}\left(I+\bar{\Sigma}_{L Z}^{i}(t)\left(E_{t}^{i}\right)^{T}\left(\widetilde{M}_{L Z, t}^{i}\right)^{-1} E_{t}^{i}\right. \\
& \left.\quad \times\left(I+\left(P(t)-\bar{\Sigma}_{L Z}^{i}(t)\right)\left(E_{t}^{i}\right)^{T}\left(\widetilde{M}_{L Z, t}^{i}\right)^{-1} E_{t}^{i}\right)^{-1}\right),
\end{aligned}
$$

where $\widetilde{M}_{L Z, t}^{i}=\alpha_{t}^{-1} I-E_{t}^{i} P(t)\left(E_{t}^{i}\right)^{\mathrm{T}}$.

For the sake of simplified calculation, $\left(E_{t}^{i}\right)^{T}\left(\widetilde{M}_{L Z, t}^{i}\right)^{-1} E_{t}^{i}$ is transformed into the following equalities:

$$
\begin{aligned}
& \left(E_{t}^{i}\right)^{T}\left(\widetilde{M}_{L Z, t}^{i}\right)^{-1} E_{t}^{i}=\left(E_{t}^{i}\right)^{T}\left(M_{L Z, t}^{i}\right)^{-1} E_{t}^{i} \times(I \\
& -\left(\left(\bar{\Sigma}_{L Z}^{i}(t)-P(t)\right)^{-1}+\left(E_{t}^{i}\right)^{T}\left(M_{L Z, t}^{i}\right)^{-1} E_{t}^{i}\right)^{-1} \\
& \left.\cdot\left(E_{t}^{i}\right)^{T}\left(M_{L Z, t}^{i}\right)^{-1} E_{t}^{i}\right)=\left(I-\left(E_{t}^{i}\right)^{T}\left(M_{L Z, t}^{i}\right)^{-1}\right. \\
& \cdot E_{t}^{i}\left(\left(\bar{\Sigma}_{L Z}^{i}(t)-P(t)\right)^{-1}\right. \\
& \left.\left.+\left(E_{t}^{i}\right)^{T}\left(M_{L Z, t}^{i}\right)^{-1} E_{t}^{i}\right)^{-1}\right) \times\left(E_{t}^{i}\right)^{T}\left(M_{L Z, t}^{i}\right)^{-1} E_{t}^{i},
\end{aligned}
$$

where $M_{L Z, t}^{i}=\alpha_{t}^{-1} I-E_{t}^{i} \bar{\Sigma}_{L Z}^{i}(t)\left(E_{t}^{i}\right)^{T}$. Using the matrix inverse lemma, $\left(E_{t}^{i}\right)^{T}\left(\widetilde{M}_{L Z, t}^{i}\right)^{-1} E_{t}^{i}$ is replaced by the equivalent forms:

$$
\begin{aligned}
& \left(E_{t}^{i}\right)^{T}\left(\widetilde{M}_{L Z, t}^{i}\right)^{-1} E_{t}^{i}=\left(E_{t}^{i}\right)^{T}\left(M_{L Z, t}^{i}\right)^{-1} \\
& \cdot E_{t}^{i}\left(I+\left(\bar{\Sigma}_{L Z}^{i}(t)-P(t)\right)\left(E_{t}^{i}\right)^{T}\left(M_{L Z, t}^{i}\right)^{-1} E_{t}^{i}\right)^{-1} \\
& =\left(I+\left(E_{t}^{i}\right)^{T}\left(M_{L Z, t}^{i}\right)^{-1} E_{t}^{i}\left(\bar{\Sigma}_{L Z}^{i}(t)-P(t)\right)\right)^{-1} \\
& \cdot\left(E_{t}^{i}\right)^{T}\left(M_{L Z, t}^{i}\right)^{-1} E_{t}^{i} .
\end{aligned}
$$

Next, $\left(I+\left(P(t)-\bar{\Sigma}_{L Z}^{i}(t)\right)\left(E_{t}^{i}\right)^{T}\left(\widetilde{M}_{L Z, t}^{i}\right)^{-1} E_{t}^{i}\right)^{-1}$ in (A.6) is calculated from (A.7) and (A.8), i.e.,

$$
\begin{aligned}
(I & \left.+\left(P(t)-\bar{\Sigma}_{L Z}^{i}(t)\right)\left(E_{t}^{i}\right)^{T}\left(\widetilde{M}_{L Z, t}^{i}\right)^{-1} E_{t}^{i}\right)^{-1} \\
& =I+\left(\bar{\Sigma}_{L Z}^{i}(t)-P(t)\right)\left(E_{t}^{i}\right)^{T}\left(M_{L Z, t}^{i}\right)^{-1} E_{t}^{i} .
\end{aligned}
$$

Summarizing the above analysis and substituting (A.8)(A.9) into (A.6), $\widehat{C}_{L Z, t}^{i}$ is determined as follows:

$$
\widehat{C}_{L Z, t}^{i}=C_{t}^{i}\left(I+\bar{\Sigma}_{L Z}^{i}(t)\left(E_{t}^{i}\right)^{T}\left(M_{L Z, t}^{i}\right)^{-1} E_{t}^{i}\right)
$$

Finally, similar to the derivation of $\widehat{C}_{L Z, t}^{i}$, filter parameters $K_{L Z, t}^{i}, \widehat{A}_{L Z, t}^{i}$, and $L_{L Z, t}^{i}$ are probed.

Step 2 (calculate the covariance matrix $\bar{\Theta}_{L Z}^{i}(t), \bar{\Sigma}_{L Z}^{i}(t+1)$, and $P(t+1))$. Theorem 5 defines the $\Theta_{L Z}^{i}(t)$ and $\Sigma_{L Z}^{i}(t+1)$. The upper bound of filtering and prediction error covariance matrices $\bar{\Theta}_{L Z}^{i}(t)$ and $\bar{\Sigma}_{L Z}^{i}(t+1)$ are derived as follows:

$$
\begin{aligned}
& \bar{\Theta}_{L Z}^{i}(t)=\left[\begin{array}{ll}
I & 0
\end{array}\right] \Theta_{L Z}^{i}(t)\left[\begin{array}{l}
I \\
0
\end{array}\right]=\left(I-K_{L Z, t}^{i} C_{t}^{i}\right) \bar{\Sigma}_{L Z}^{i}(t) \\
& \cdot\left(I-K_{L Z, t}^{i} C_{t}^{i}\right)^{T}+\left(K_{L Z, t}^{i}\left(\widehat{C}_{L Z, t}^{i}-C_{t}^{i}\right)\right)(P(t) \\
& \left.\quad-\bar{\Sigma}_{L Z}^{i}(t)\right)\left(K_{L Z, t}^{i}\left(\widehat{C}_{L Z, t}^{i}-C_{t}^{i}\right)\right)^{T}+\left(\bar{\Sigma}_{L Z}^{i}(t)\right. \\
& \left.+K_{L Z, t}^{i}\left(\widehat{C}_{L Z, t}^{i}\left(P(t)-\bar{\Sigma}_{L Z}^{i}(t)\right)-C_{t}^{i} P(t)\right)\right) \\
& \quad \times\left(E_{t}^{i}\right)^{T}\left(\widetilde{M}_{L Z, t}^{i}\right)^{-1} E_{t}^{i} \times\left(\bar{\Sigma}_{L Z}^{i}(t)\right. \\
& \left.+K_{L Z, t}^{i}\left(\widehat{C}_{L Z, t}^{i}\left(P(t)-\bar{\Sigma}_{L Z}^{i}(t)\right)-C_{t}^{i} P(t)\right)\right)^{T} \\
& +\alpha_{t}^{-1} K_{L Z, t}^{i} \mathscr{H}_{t}^{i}\left(\mathscr{H}_{t}^{i}\right)^{T}\left(K_{L Z, t}^{i}\right)^{T} \\
& +K_{L Z, t}^{i} R_{t}^{i}\left(K_{L Z, t}^{i}\right)^{T},
\end{aligned}
$$

and

$$
\begin{aligned}
& \bar{\Sigma}_{L Z}^{i}(t+1)=\left[\begin{array}{ll}
I & 0
\end{array}\right] \Sigma_{L Z}^{i}(t+1)\left[\begin{array}{l}
I \\
0
\end{array}\right]=\left(A_{t}\right. \\
& \left.\quad-L_{L Z, t}^{i} C_{t}^{i}\right) \bar{\Sigma}_{L Z}^{i}(t)\left(A_{t}-L_{L Z, t}^{i} C_{t}^{i}\right)^{T}+B_{t} Q_{t} B_{t}^{T} \\
& +L_{L Z, t}^{i} R_{t}^{i}\left(L_{L Z, t}^{i}\right)^{T}+\left(A_{t}-\widehat{A}_{L Z, t}\right. \\
& \left.+L_{L Z, t}^{i}\left(\widehat{C}_{L Z, t}^{i}-C_{t}^{i}\right)\right)\left(P(t)-\bar{\Sigma}_{L Z}^{i}(t)\right) \times\left(A_{t}\right. \\
& \left.\quad-\widehat{A}_{L Z, t}^{i}+L_{L Z, t}^{i}\left(\widehat{C}_{L Z, t}^{i}-C_{t}^{i}\right)\right)^{T} \\
& +\left(\left(A_{t}-L_{L Z, t}^{i} C_{t}^{i}\right) \bar{\Sigma}_{L Z}^{i}(t)\right. \\
& +\left(A_{t}-\widehat{A}_{L Z, t}^{i}+L_{L Z, t}^{i}\left(\widehat{C}_{L Z, t}^{i}-C_{t}^{i}\right)\right) \\
& \left.\quad \times\left(P(t)-\bar{\Sigma}_{L Z}^{i}(t)\right)\right)\left(E_{t}^{i}\right)^{T}\left(\widetilde{M}_{L Z, t}^{i}\right)^{-1} E_{t}^{i}\left(\bar{\Sigma}_{L Z}^{i}(t)\right. \\
& \quad+\left(A_{t}-L_{L Z, t}^{i} C_{t}^{i}\right)^{T}+\left(P(t)-\bar{\Sigma}_{L Z}^{i}(t)\right) \\
& \left.\quad+\left(A_{t}-\widehat{A}_{L Z, t}^{i}+L_{L Z, t}^{i}\left(\widehat{C}_{L Z, t}^{i}-C_{t}^{i}\right)\right)^{T}\right)
\end{aligned}
$$




$$
\begin{aligned}
& +\alpha_{t}^{-1}\left(\mathscr{F}_{t}-L_{L Z, t}^{i} \mathscr{H}_{t}^{i}\right)\left(\mathscr{F}_{t}-L_{L Z, t}^{i} \mathscr{H}_{t}^{i}\right)^{T} \\
& -B_{t} S_{t}^{i}\left(L_{L Z, t}^{i}\right)^{T}-L_{L Z, t}^{i}\left(S_{t}^{i}\right)^{T} B_{t}^{T} .
\end{aligned}
$$

In summary, substituting filter parameters into (A.11) and (A.12), respectively, the upper bounds of estimation error covariance matrices are rewritten as

$$
\begin{aligned}
\bar{\Theta}_{L Z}^{i}(t)= & \bar{\Sigma}_{L Z}^{i}(t) \\
& +\bar{\Sigma}_{L Z}^{i}(t)\left(E_{t}^{i}\right)^{T}\left(\widetilde{M}_{L Z, t}^{i}\right)^{-1} E_{t}^{i} \bar{\Sigma}_{L Z}^{i}(t) \\
& -\Lambda_{L Z}^{i}(t)\left(\Xi_{L Z}^{i}(t)\right)^{-1}\left(\Lambda_{L Z}^{i}(t)\right)^{T},
\end{aligned}
$$

and

$$
\begin{aligned}
\bar{\Sigma}_{L Z}^{i}( & +1) \\
= & A_{t} \bar{\Sigma}_{L Z}^{i}(t)\left(I+\left(E_{t}^{i}\right)^{T}\left(M_{L Z, t}^{i}\right)^{-1} E_{t}^{i} \bar{\Sigma}_{L Z}^{i}(t)\right) A_{t}^{T} \\
& +B_{t} Q_{t} B_{t}^{T}+\alpha_{t}^{-1} \mathscr{F}_{t} \mathscr{F}_{t}^{T} \\
& -\Delta_{L Z}^{i}(t)\left(\Xi_{L Z}^{i}(t)\right)^{-1}\left(\Delta_{L Z}^{i}(t)\right)^{T} .
\end{aligned}
$$

Furthermore, the state covariance with time-varying parametric uncertainties is defined as

$$
\begin{aligned}
\widetilde{P}(t+1)= & E\left(x(t+1) x^{T}(t+1)\right) \\
= & \left(A_{t}+\mathscr{F}_{t} F_{t} E_{t}\right) \widetilde{P}(t)\left(A_{t}+\mathscr{F}_{t} F_{t} E_{t}\right)^{T} \\
& +B_{t} Q_{t} B_{t}^{T} .
\end{aligned}
$$

Following the upper bound, the state covariance matrix is obtained as

$$
\begin{aligned}
\widetilde{P}(t+1)= & \left(A_{t}+\mathscr{F}_{t} F_{t} E_{t}\right) P(t)\left(A_{t}+\mathscr{F}_{t} F_{t} E_{t}\right)^{T} \\
& +B_{t} Q_{t} B_{t}^{T} \\
\leqslant & A_{t}\left(P^{-1}(t)-\alpha_{t} E_{t}^{T} E_{t}\right)^{-1} A_{t}^{T} \\
& +\alpha_{t}^{-1} \mathscr{F}_{t}{ }_{t}{ }_{t}^{T}+B_{t} Q_{t} B_{t}^{T} \\
= & A_{t} P(t) A_{t}^{T}+A_{t} P(t) E_{t}^{T} \widetilde{M}_{t}^{-1} E_{t} P(t) A_{t}^{T} \\
& +\alpha_{t}^{-1} \mathscr{F}_{t} \mathscr{F}_{t}^{T}+B_{t} Q_{t} B_{t}^{T}=P(t+1),
\end{aligned}
$$

with the initial value $P(0)=x(0) x^{T}(0)+P_{0}$, which is similarly calculated in [11].

\section{Data Availability}

The results of a numerical example are demonstrated to illustrate the effectiveness of the proposed distributed fusion estimation strategy. The considered target tracking systems with intermittent measurements are uncertain systems, which contains three sensors.

\section{Conflicts of Interest}

The authors declare that there are no conflicts of interest regarding the publication of this paper.

\section{Acknowledgments}

This work was supported by the Natural Science Foundation of China $(61877065,61873117,61772253,61633016$, and 61472172).

\section{References}

[1] S. Sun and G. Wang, "Modeling and estimation for networked systems with multiple random transmission delays and packet losses," Systems \& Control Letters, vol. 73, pp. 6-16, 2014.

[2] Y.-L. Wang and Q.-L. Han, "Modelling and observer-based Ho controller design for networked control systems," IET Control Theory \& Applications, vol. 8, no. 15, pp. 1478-1486, 2014.

[3] P. Kachroo and S. Sastry, "Traffic Assignment Using a DensityBased Travel-Time Function for Intelligent Transportation Systems," IEEE Transactions on Intelligent Transportation Systems, vol. 17, no. 5, pp. 1438-1447, 2016.

[4] M. Wu, L. Tan, and N. Xiong, "Data prediction, compression, and recovery in clustered wireless sensor networks for environmental monitoring applications," Information Sciences, vol. 329, pp. 800-818, 2016.

[5] D. Dolz, I. Penarrocha, and R. Sanchis, "Performance tradeoffs for networked jump observer-based fault diagnosis," IEEE Transactions on Signal Processing, vol. 63, no. 10, pp. 2692-2703, 2015.

[6] L. Yu, T. Jiang, and Y. Cao, "Energy cost minimization for distributed internet data centers in smart microgrids considering power outages," IEEE Transactions on Parallel and Distributed Systems, vol. 26, no. 1, pp. 120-130, 2015.

[7] X. Ge and Q.-L. Han, "Distributed event-triggered Hoo filtering over sensor networks with communication delays," Information Sciences, vol. 291, pp. 128-142, 2015.

[8] Q. Liu, Z. Wang, X. He, and D. H. Zhou, "Event-based recursive distributed filtering over wireless sensor networks," Institute of Electrical and Electronics Engineers Transactions on Automatic Control, vol. 60, no. 9, pp. 2470-2475, 2015.

[9] J. Hu, Z. Wang, D. Chen, and F. E. Alsaadi, "Estimation, filtering and fusion for networked systems with networkinduced phenomena: new progress and prospects," Information Fusion, vol. 31, pp. 65-75, 2016.

[10] T. Tian, S. Sun, and N. Li, "Multi-sensor information fusion estimators for stochastic uncertain systems with correlated noises," Information Fusion, vol. 27, pp. 126-137, 2016.

[11] J. Feng, Z. Wang, and M. Zeng, "Distributed weighted robust Kalman filter fusion for uncertain systems with autocorrelated and cross-correlated noises," Information Fusion, vol. 14, no. 1, pp. 78-86, 2013.

[12] Y. Liu, X. He, Z. Wang, and D. Zhou, "Optimal filtering for networked systems with stochastic sensor gain degradation," Automatica, vol. 50, no. 5, pp. 1521-1525, 2014.

[13] S. Wang, H. Fang, and X. Tian, "Minimum variance estimation for linear uncertain systems with one-step correlated noises and incomplete measurements," Digital Signal Processing, vol. 49, pp. 126-136, 2016. 
[14] J. Feng and M. Zeng, "Optimal distributed Kalman filtering fusion for a linear dynamic system with cross-correlated noises," International Journal of Systems Science, vol. 43, no. 2, pp. 385398, 2012.

[15] L. Yan, X. Rong Li, Y. Xia, and M. Fu, "Optimal sequential and distributed fusion for state estimation in cross-correlated noise," Automatica, vol. 49, no. 12, pp. 3607-3612, 2013.

[16] Xue-wen Chen and Yue Zhou, "Modelling and Analysis of Automobile Vibration System Based on Fuzzy Theory under Different Road Excitation Information," Complexity, vol. 2018, Article ID 2381568, 9 pages, 2018.

[17] L. Liu, A. Yang, X. Tu, M. Fei, and W. Naeem, "Distributed weighted fusion estimation for uncertain networked systems with transmission time-delay and cross-correlated noises," $\mathrm{Neu}$ rocomputing, vol. 270, pp. 54-65, 2017.

[18] J. Na, M. N. Mahyuddin, G. Herrmann, X. Ren, and P. Barber, "Robust adaptive finite-time parameter estimation and control for robotic systems," International Journal of Robust and Nonlinear Control, vol. 25, no. 16, pp. 3045-3071, 2015.

[19] J. Na, Y. Huang, X. Wu, G. Gao, G. Herrmann, and J. Z. Jiang, "Active adaptive estimation and control for vehicle suspensions with prescribed performance," IEEE Transactions on Control Systems Technology, pp. 1-15, 2017.

[20] C. Zhang and Y. Jia, "Distributed Kalman consensus filter with event-triggered communication: formulation and stability analysis," Journal of The Franklin Institute, vol. 354, no. 13, pp. 5486-5502, 2017.

[21] Z. Wang, H. Dong, B. Shen, and H. Gao, "Finite-horizon Ho filtering with missing measurements and quantization effects," IEEE Transactions on Automatic Control, vol. 58, no. 7, pp. 17071718, 2013.

[22] Z. Yang, K. Li, Q. Niu, and Y. Xue, "A comprehensive study of economic unit commitment of power systems integrating various renewable generations and plug-in electric vehicles," Energy Conversion and Management, vol. 132, pp. 460-481, 2017.

[23] H. Rezaei, R. M. Esfanjani, and M. Farsi, "Robust filtering for uncertain networked systems with randomly delayed and lost measurements," IET Signal Processing, vol. 9, no. 4, pp. 320-327, 2015.

[24] H. Rezaei, M. Reza, and M. H. Sedaaghi, "Improved robust finite-horizon Kalman filtering for uncertain networked timevarying systems," Information Sciences, vol. 293, pp. 263-274, 2015.

[25] B. Chen, W.-A. Zhang, and L. Yu, "Distributed finite-horizon fusion Kalman filtering for bandwidth and energy constrained wireless sensor networks," IEEE Transactions on Signal Processing, vol. 62, no. 4, pp. 797-812, 2014.

[26] B. Chen, G. Hu, D. W. C. Ho, W.-A. Zhang, and L. Yu, "Distributed Robust Fusion Estimation with Application to State Monitoring Systems," IEEE Transactions on Systems, Man, and Cybernetics: Systems, vol. 47, no. 11, pp. 2994-3005, 2017.

[27] T. La-inchua, P. Niamsup, and Xinzh Liu I, "Finite-Time Stability of Large-Scale Systems with Interval Time-Varying Delay in Interconnection," Complexity, vol. 2017, Article ID 1972748, 11 pages, 2017.

[28] S. Wang, H. Fang, and X. Tian, "Recursive estimation for nonlinear stochastic systems with multi-step transmission delays, multiple packet dropouts and correlated noises," Signal Processing, vol. 115, pp. 164-175, 2015.

[29] B. Chen, W.-A. Zhang, and L. Yu, "Distributed fusion estimation with missing measurements, random transmission delays and packet dropouts," IEEE Transactions on Automatic Control, vol. 59, no. 7, pp. 1961-1967, 2014.

[30] B. Chen, W. Zhang, G. Hu, and L. Yu, "Networked fusion Kalman filtering with multiple uncertainties," IEEE Transactions on Aerospace and Electronic Systems, vol. 51, no. 3, pp. 2332-2349, 2015.

[31] M. J. García-Ligero, A. Hermoso-Carazo, and J. Linares-Pérez, "Distributed fusion estimation in networked systems with uncertain observations and Markovian random delays," Signal Processing, vol. 106, pp. 114-122, 2015.

[32] S. Sun and W. Xiao, "Optimal linear estimators for systems with multiple random measurement delays and packet dropouts," International Journal of Systems Science, vol. 44, no. 2, pp. 358370, 2013.

[33] D. Brizhinev, N. Ryan, and R. Bradbury, "Modelling Hegemonic Power Transition in Cyberspace," Complexity, vol. 2018, Article ID 9306128, 13 pages, 2018.

[34] G. Miao, J. Cao, A. Alsaedi, and F. E. Alsaadi, "Event-triggered containment control for multi-agent systems with constant time delays," Journal of The Franklin Institute, vol. 354, no. 15, pp. 6956-6977, 2017.

[35] T.-F. Li and J. Fu, "Event-triggered control of switched linear systems," Journal of The Franklin Institute, vol. 354, no. 15, pp. 6451-6462, 2017.

[36] A. Farnam and R. Mahboobi Esfanjani, "Improved stabilization method for networked control systems with variable transmission delays and packet dropout," ISA Transactions ${ }^{\circledR}$, vol. 53, no. 6, pp. 1746-1753, 2014.

[37] G. Guo, L. Ding, and Q.-L. Han, “A distributed event-triggered transmission strategy for sampled-data consensus of multiagent systems," Automatica, vol. 50, no. 5, pp. 1489-1496, 2014.

[38] C. Peng, M.-R. Fei, E. Tian, and Y.-P. Guan, "On hold or drop out-of-order packets in networked control systems," Information Sciences, vol. 268, pp. 436-446, 2014.

[39] X.-M. Zhang and Q.-L. Han, "Network-based $H_{\infty}$ filtering using a logic jumping-like trigger," Automatica, vol. 49, no. 5, pp. 1428-1435, 2013.

[40] C. Peng, Q.-L. Han, and D. Yue, "To transmit or not to transmit: a discrete event-triggered communication scheme for networked takagi-sugeno fuzzy systems," IEEE Transactions on Fuzzy Systems, vol. 21, no. 1, pp. 164-170, 2013.

[41] H. Wang, P. Shi, C.-C. Lim, and Q. Xue, "Event-triggered control for networked Markovian jump systems," International Journal of Robust and Nonlinear Control, vol. 25, no. 17, pp. 3422-3438, 2015.

[42] J. Na, A. S. Chen, G. Herrmann, R. Burke, and C. Brace, "Vehicle Engine Torque Estimation via Unknown Input Observer and Adaptive Parameter Estimation," IEEE Transactions on Vehicular Technology, vol. 67, no. 1, pp. 409-422, 2018.

[43] F. Yang, Z. Wang, G. Feng, and X. Liu, "Robust filtering with randomly varying sensor delay: the finite-horizon case," IEEE Transactions on Circuits and Systems I: Regular Papers, vol. 56, no. 3, pp. 664-672, 2009.

[44] Z. Yang, K. Li, Q. Niu, and Y. Xue, "A novel parallel-series hybrid meta-heuristic method for solving a hybrid unit commitment problem," Knowledge-Based Systems, vol. 134, pp. 13-30, 2017.

[45] P. Zhang, W. Qi, and Z. Deng, "Hierarchical fusion robust Kalman filter for clustering sensor network time-varying systems with uncertain noise variances," International Journal of Adaptive Control and Signal Processing, vol. 29, no. 1, pp. 99-122, 2015. 


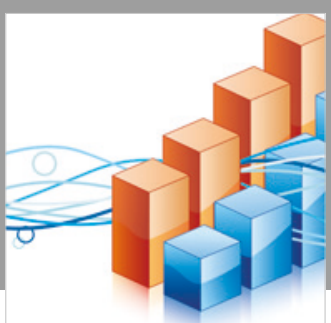

Advances in

Operations Research

\section{-n-m}
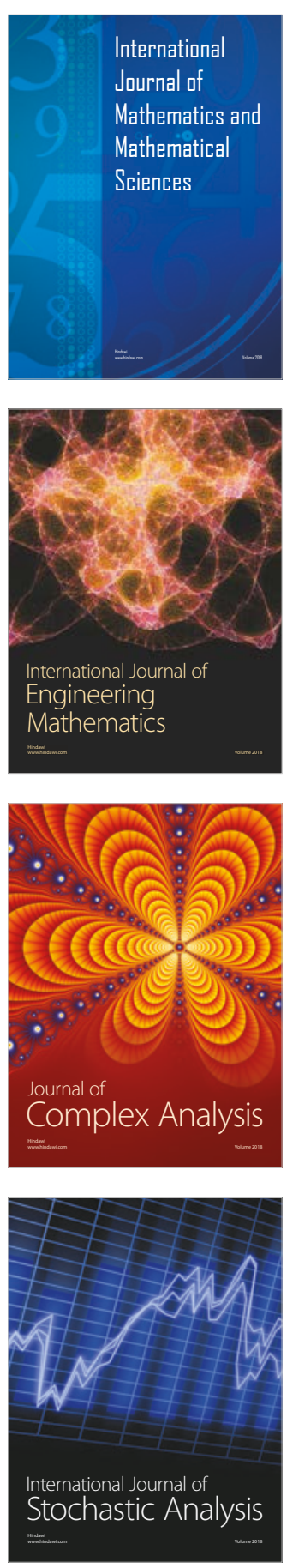
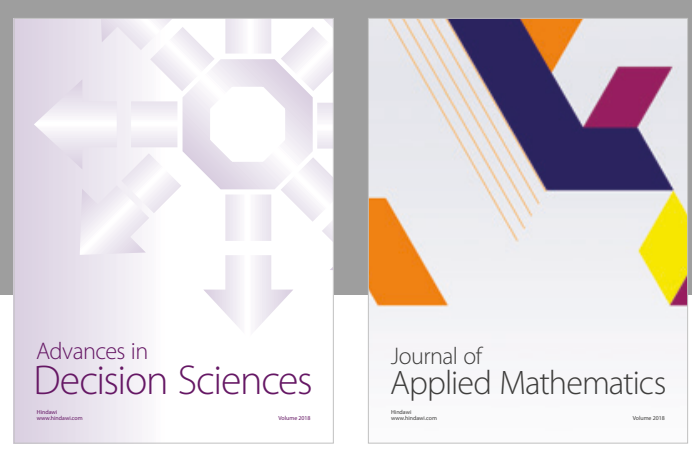

Journal of

Applied Mathematics
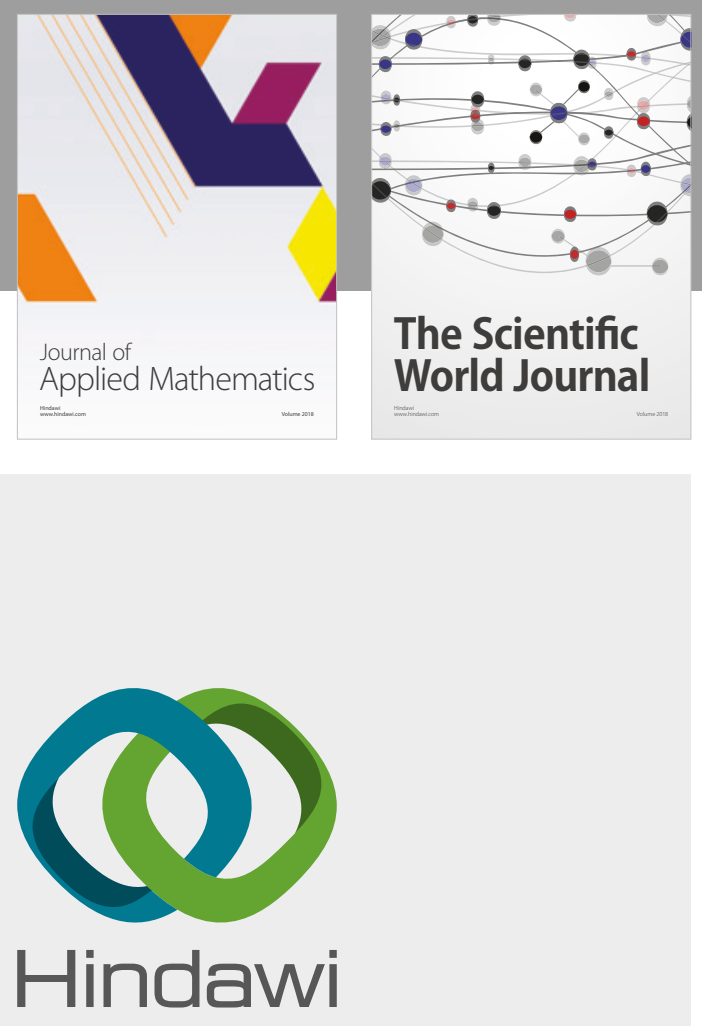

Submit your manuscripts at

www.hindawi.com

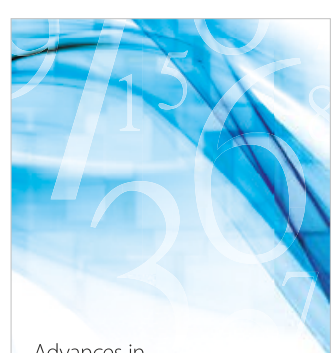

Advances in
Numerical Analysis
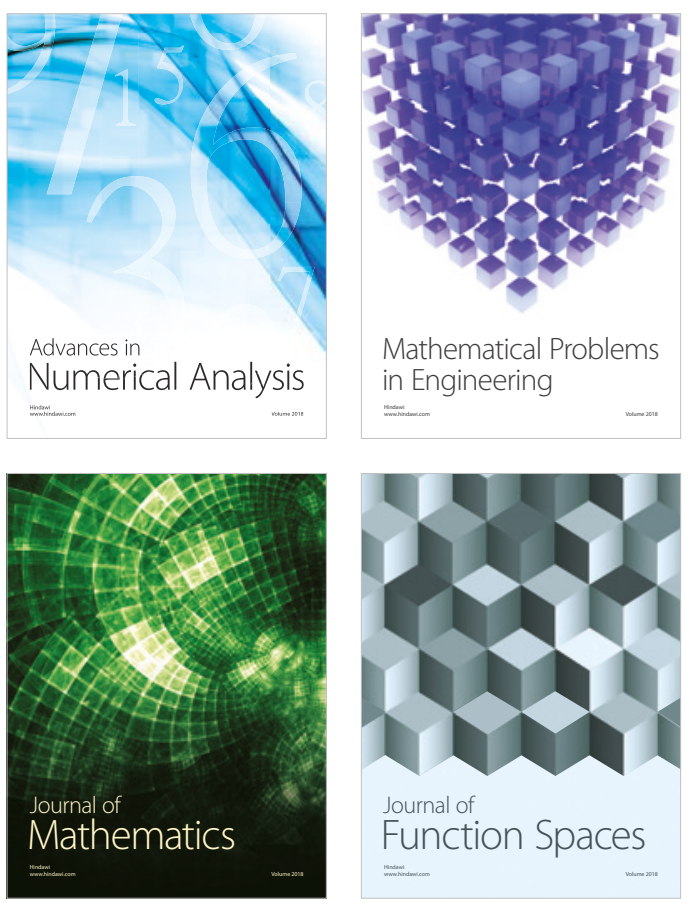

Mathematical Problems in Engineering

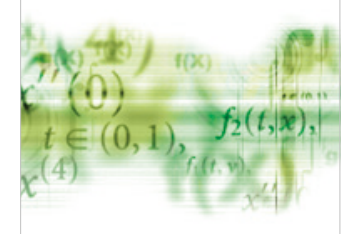

International Journal of

Differential Equations

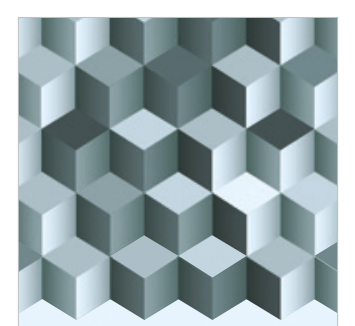

Journal of

Function Spaces
The Scientific

World Journal

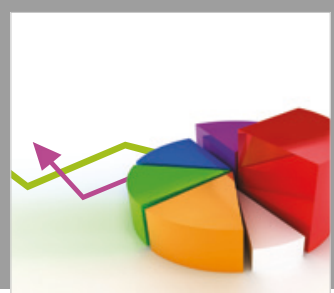

Journal of

Probability and Statistics
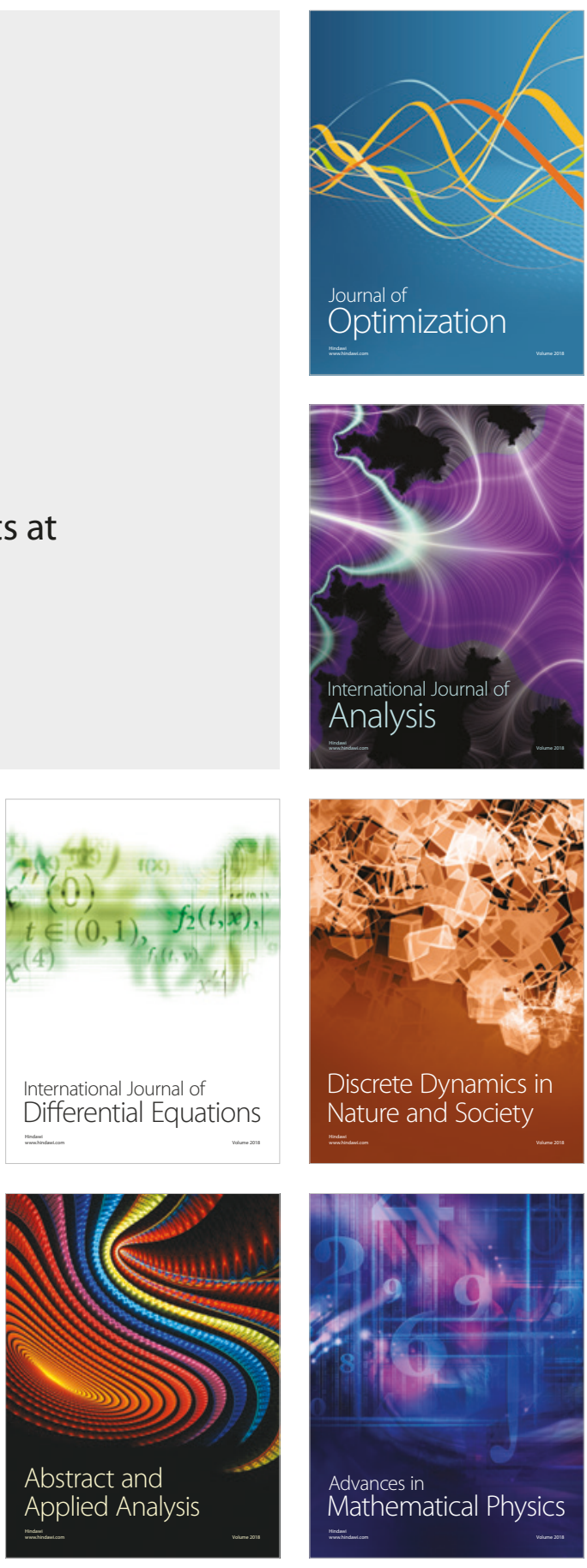